\title{
Two-Channel Perfect-Reconstruction FIR QMF Structures Which Yield Linear-Phase Analysis and Synthesis Filters
}

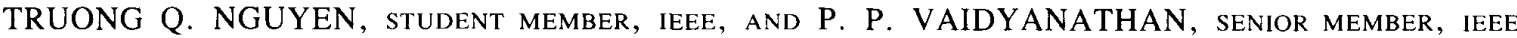

\begin{abstract}
Among all cases of two-channel FIR perfect-reconstruction QMF structures which yield linear-phase filters, only two cases yield good filters in the practical sense. In this paper, lattice-type structures are derived for both cases-to structurally enforce the perfectreconstruction and linear-phase properties simultaneously. The structure in the first case is related to the linear prediction lattice structure. For the second case, new structures are developed by propagating the perfect-reconstruction and linear phase properties. Design examples, based on optimization of the parameters in the lattice structures, are presented for both cases.
\end{abstract}

\section{INTRODUCTION}

$\mathrm{Q}$ UADRATURE mirror filters (in short, QMF's) are used in many speech and communications applications [1], [2]. A two-channel QMF bank is shown in Fig. 1 where $H_{0}(z), H_{1}(z)$ are the transfer functions of analysis bank filters, and $F_{0}(z), F_{1}(z)$ are the synthesis filters. The reconstructed signal, in general, suffers from aliasing error, amplitude, and phase distortions, due to the fact that the filters $H_{0}(z), H_{1}(z), F_{0}(z)$, and $F_{1}(z)$ are not ideal. A common requirement in most applications is that the reconstructed signal $\hat{x}(n)$ should be "as close" to $x(n)$ as possible in some well-defined sense. A system that is free from aliasing, amplitude, and phase distortions is called a perfect-reconstruction QMF bank. The reconstructed signal is therefore just a time-delayed version of the transmitted signal $x(n)$, i.e., $y(n)=b x\left(n-n_{0}\right)$ for some nonzero constant $b$ and some positive integer $n_{0}$.

The theory of perfect reconstruction, when the number of channels is a power of 2, is well known [2]-[4]. The design method in [2] and [3] is based on spectral factorization of an FIR halfband filter. The elegant choices of $H_{0}(z), H_{1}(z), F_{0}(z)$, and $F_{1}(z)$ cancel aliasing and yield a perfect-reconstruction system. Some methods of perfect reconstruction for an arbitrary number of channels have been reported recently [5]-[7]. In [6] and [10], a procedure for designing two-channel perfect-reconstruction systems with linear-phase FIR filters has been outlined. This procedure is based on judicious factorization of a

Manuscript received April 23, 1988; revised September 26, 1988. This work was supported in part by the National Science Foundation under Grants DCl 8552579 and MIP 8604456. T. Q. Nguyen was an Aerojet Fellow during the first half of the period when this work was performed.

The authors are with the Department of Electrical Engineering, California Institute of Technology, Pasadena, CA 91125.

IEEE Log Number 8926679.

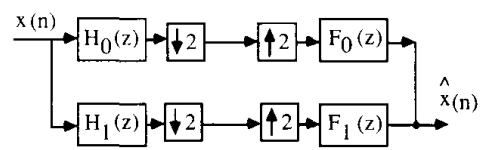

Fig. 1. The 2-channel QMF bank.

linear-phase FIR halfband filter. However, the number of possible spectral factors grows exponentially with respect to the order of the filters and the resulting filters are not guaranteed to be optimal. In a recent paper [20], the authors discussed several properties of $M$-channel linearphase perfect-reconstruction systems and, moreover, they derived a lattice structure for the two-channel linear-phase perfect-reconstruction QMF bank. Based on optimization of these lattice coefficients, they have been able to obtain good design examples.

The linear-phase analysis filters $H_{0}(z)$ and $H_{1}(z)$ of the two-channel perfect-reconstruction systems developed in [6], [10], and [20] have odd order and opposite symmetry. In other words, the impulse responses of $H_{0}(z)$ and $H_{1}(z)$ are symmetric and antisymmetric, respectively, or vice versa. This, as we shall show in Section II, is one of the two cases that yield nontrivial perfect-reconstruction linear-phase FIR filters. The other choice yields filters with even orders and with symmetric impulse responses.

Let $H_{k}(z)$ and $F_{k}(z), 0 \leq k \leq 1$, denote the analysis and synthesis filters, respectively, of a two-channel QMF bank. We can always represent them in terms of their polyphase components [1], [5], [11] as

$$
\begin{aligned}
& H_{k}(z)=\sum_{l=0}^{1} z^{-l} E_{k, l}\left(z^{2}\right) \quad \text { and } \\
& F_{k}(z)=\sum_{l=0}^{1} z^{-(1-l)} R_{l, k}\left(z^{2}\right) .
\end{aligned}
$$

With $\boldsymbol{E}(z) \triangleq\left[E_{k, l}(z)\right]$ and $\boldsymbol{R}(z) \triangleq\left[\boldsymbol{R}_{l, k}(z)\right]$, we can redraw the QMF bank of Fig. 1 as Fig. 2. Based on standard identities for multirate systems [1], Fig. 2 can be redrawn as Fig. 3. We can obtain a perfect-reconstruction system [5] if we choose $R(z)=E^{-1}(z)$. If the analysis filters are FIR, the choice $\boldsymbol{R}(z)=\boldsymbol{E}^{-1}(z)$ gives rise to FIR synthesis filters, provided det $\boldsymbol{E}(z)=b z^{-r}$ where $b$ and $r$ are a nonzero constant and a nonnegative integer, 


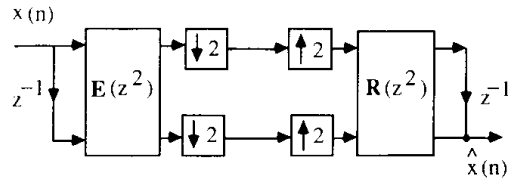

Fig. 2. An equivalent structure for Fig. 1.

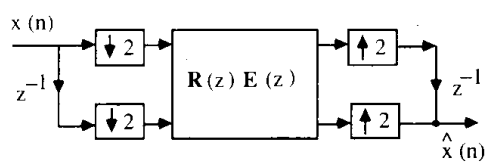

Fig. 3. A redrawing of Fig. 2.

respectively. In this paper, the term " perfect reconstruction" is synonymous with the condition $\operatorname{det} \boldsymbol{E}(z)=b z^{-r}$.

Our aim is to obtain a structure for the pair of transfer functions $\left[H_{0}(z), H_{1}(z)\right]$ with overall form as in Fig. 4 . The structure should have the following features regardless of the multiplier values in the structure:

1) $H_{0}(z)$ and $H_{1}(z)$ are linear-phase FIR filters;

2) $\operatorname{det} \boldsymbol{E}(z)=b z^{-r}$;

3) every pair of FIR transfer functions $\left[H_{0}(z), H_{1}(z)\right]$ with the above two properties should be realizable by the structure.

Such a structure will be called a linear-phase perfectreconstruction structure, and such a pair $\left[H_{0}(z), H_{1}(z)\right]$ will be called a perfect-reconstruction pair. As a first step toward this goal, we obtain structures satisfying Property 1 and then incorporate Property 2 above.

Most of the two-channel QMF designs, known so far, satisfy the power-complementary property (i.e., $\left.\left|H_{0}\left(e^{j \omega}\right)\right|^{2}+\left|H_{1}\left(e^{j \omega}\right)\right|^{2}=1\right)$ either approximately, as in [1] and [16], or exactly as in perfect-reconstruction systems [2]-[5]. This condition, however, is not necessary for perfect reconstruction [6], [20]. In fact, if we constrain $H_{0}(z)$ and $H_{1}(z)$ to be linear-phase filters, it will be necessary to give up the power-complementary property [21]. All the QMF analysis filter designs in this paper have the linear-phase and perfect-reconstruction property, accomplished by relaxing the power-complementary requirement.

We would like to mention the fact that for the case of $M$ channels where $M>2$, all three requirements, namely, perfect reconstruction, linear phase, and power complementarity, can be simultaneously imposed on the QMF structure (see comments below (9) in [20]). It turns out that the resulting structure in [20] yields only trivial filters for the case of two-channel QMF bank [21].

In Section II, we derive the necessary form of $\boldsymbol{E}(z)$ such that $H_{0}(z)$ and $H_{1}(z)$ are linear-phase FIR analysis filters of orders $N_{0}-1$ and $N_{1}-1$, respectively. Imposing the perfect-reconstruction condition on the above $\boldsymbol{E}(z)$, we first show that the only two cases which yield nontrivial analysis filters are such that the sum of their lengths is a multiple of 4 and either

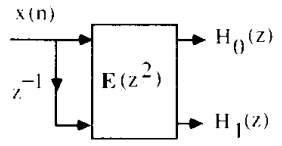

Fig. 4. Pertaining to the discussion of a perfect-reconstruction pair

1) both filters have odd order and opposite symmetry, or

2) both filters have even order and are symmetric.

We study the first case extensively in Section III and present a structure which covers all pairs of odd-order linear-phase FIR analysis filters with opposite symmetry. ${ }^{1}$ This structure is closely related to the linear prediction lattice structure [8]. With the perfect-reconstruction condition imposed on this lattice structure (with some modifications), most pairs of linear-phase perfect-reconstruction odd-order analysis filters (with opposite symmetry) are covered. We follow the theory by a design example. Similarly, the second case is discussed in Section IV. Based on the theory of symmetric polynomials, we show that all symmetric even-order linear-phase perfect-reconstruction pairs can be realized using this structure. A design example is included.

Notations Used in the Paper: We consider only realcoefficient linear-phase FIR filters in this paper. Boldfaced letters indicate vectors and matrices whereas superscript $T$ denotes transposition. Node-labelings, such as $T_{N}(z)$ in Fig. 5, are often used to denote the transfer function from the input $x(n)$ to the indicated node. If the impulse response $t_{n}$ of $T(z)$ is symmetric, we say " $T(z)$ is symmetric,"' and so on. The center of a linear-phase FIR transfer function is defined to be the center of symmetry or antisymmetry of $t_{n}$. Clearly, the center of $T(z)$ could be either an integer or an odd multiple of $(1 / 2)$. The tilde accent on a transfer matrix $\boldsymbol{F}(z)$ is defined such that $\tilde{\boldsymbol{F}}(z)=\boldsymbol{F}^{T}\left(z^{-1}\right)$. In the scalar case, $\tilde{\boldsymbol{F}}(z)=F\left(z^{-1}\right)$. The mirror image of $T(z)$, denoted by $\hat{T}(z)$ is defined as $\hat{T}(z) \triangleq z^{-(N-1)} T\left(z^{-1}\right)$ where $N-1$ is the degree of $T(z)$. $[T(z), U(z)]$ is called a mirror image pair (in short, MIP) if $T(z)=\hat{U}(z)$. Naturally, two transfer functions in an MIP have the same order. Similarly, for a transfer matrix $\boldsymbol{E}(z), \hat{\boldsymbol{E}}(z)$ is defined to be a matrix whose elements are the mirror images of the corresponding element of $\boldsymbol{E}(z)$, i.e., $\hat{\boldsymbol{E}}(z)=\left[\hat{E}_{k, l}(z)\right]$. For brevity, "linear-phase" and "perfect-reconstruction" are abbreviated as LP and PR, respectively. The adjectives, symmetric, antisymmetric, even, and odd are also abbreviated as S, A, E, and $\mathrm{O}$, respectively. For example, if $H_{0}(z)$ is symmetric, even order and $H_{1}(z)$ is antisymmetric, odd order, then the pair [ $\left.H_{0}(z), H_{1}(z)\right]$ is called an SAEO pair.

At this point, we would like to warn the reader that the acronym QMF is a misnomer. The letter " $M$ ' which stands for "mirror" was originally invented [24] because the response $\left|H_{1}\left(e^{j \omega}\right)\right|$ was a perfect mirror image of $\left|H_{0}\left(e^{j \omega}\right)\right|$ with respect to $2 \pi / 4$ which is a "quarter" of

\footnotetext{
'Independent work in this direction has also been reported in [25]
} 


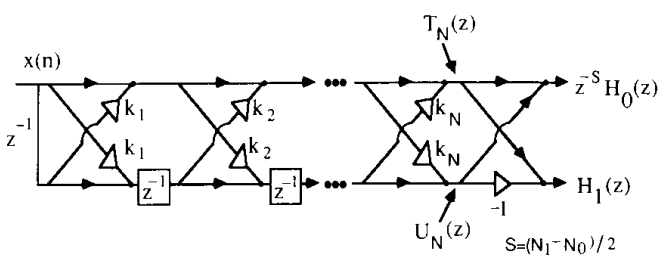

Fig. 5. The $2 \times 2$ LPC lattice structure and its resulting SA LP pair $\left[H_{0}(z), H_{\mathrm{b}}(z)\right]$

$2 \pi$. In order to obtain linear-phase analysis filters which are compatible with perfect reconstruction, it is necessary to give up the "Quadrature Mirror" property. However, the better alternative to the acronym QMF, viz., "maximally decimated analysis/synthesis system," is too inconvenient for frequent use (unless a new acronym such as MDASS is added to the evergrowing list of acronyms!). We shall therefore use the term QMF to signify any system which can be drawn as in Fig. 1 .

\section{Two-Channel LP PR FIR QMF Banks}

The impulse response of a linear-phase real-coefficient filter could be either a symmetric or an antisymmetric sequence and, furthermore, its center could be either an integer or an odd multiple of ( $1 / 2$ ) (i.e., its order could be either even or odd [9]). As recognized by Vetterli in [10], there are thus several cases to study. With two analysis filters $H_{0}(z)$ and $H_{1}(z)$ to consider, we have in total 16 cases. Let the centers and orders of $H_{k}(z)$ be $c_{k}$ and $N_{k}-$ 1 , respectively, $0 \leq k \leq 1$. Since $H_{k}(z), 0 \leq k \leq 1$ is linear phase, it satisfies

$$
\tilde{H}_{k}(z)=J_{k} z^{2 c_{k}} H_{k}(z)
$$

where

$$
J_{k}=\left\{\begin{aligned}
1, & H_{k}(z) \text { is symmetric } \\
-1, & H_{k}(z) \text { is antisymmetric }
\end{aligned}\right.
$$

In terms of the polyphase components $E_{k, l}(z), H_{k}(z)$ is represented as

$$
\left[\begin{array}{l}
H_{0}(z) \\
H_{1}(z)
\end{array}\right]=E\left(z^{2}\right)\left[\begin{array}{l}
1 \\
z^{-1}
\end{array}\right]
$$

where

$$
\boldsymbol{E}\left(z^{2}\right)=\left[\begin{array}{cc}
E_{0,0}\left(z^{2}\right) & E_{0,1}\left(z^{2}\right) \\
E_{1,0}\left(z^{2}\right) & E_{1,1}\left(z^{2}\right)
\end{array}\right] .
$$

Using (3) in (2), we obtain

$$
\begin{aligned}
& {\left[\tilde{E}_{k, 0}\left(z^{2}\right) \tilde{E}_{k, 1}\left(z^{2}\right)\right]\left[\begin{array}{l}
1 \\
z
\end{array}\right]} \\
& \quad=z^{2 c k} J_{k}\left[E_{k, 0}\left(z^{2}\right) E_{k, 1}\left(z^{2}\right)\right]\left[\begin{array}{c}
1 \\
z^{-1}
\end{array}\right] ; \quad 0 \leq k \leq 1 .
\end{aligned}
$$

We shall define the center of $E_{k, j}(z)$ as $c_{k j}$ where

$$
c_{k 0} \triangleq\left\{\begin{array}{l}
c_{k} / 2, \quad \text { if } c_{k} \text { is an integer; } \\
c_{k} / 2-1 / 4, \\
\quad \text { if } c_{k} \text { is an odd multiple of }(1 / 2) .
\end{array}\right.
$$

$$
c_{k 1} \triangleq\left\{\begin{array}{l}
c_{k} / 2-1 / 2, \quad \text { if } c_{k} \text { is an integer; } \\
c_{k} / 2-1 / 4, \\
\quad \text { if } c_{k} \text { is an odd multiple of }(1 / 2) .
\end{array}\right.
$$

Using (6a) and (6b) in (5) and comparing like powers of both sides, we have the necessary form of $\boldsymbol{E}(z)$ which yields linear-phase analysis filters. Thus,

$$
\left[\begin{array}{c}
\tilde{E}_{k, 0}(z) \\
\tilde{E}_{k, 1}(z)
\end{array}\right]=\left\{\begin{array}{c}
J_{k}\left[\begin{array}{c}
z^{2 c k 0} E_{k, 0}(z) \\
z^{2 c k} E_{k, 1}(z)
\end{array}\right], \\
\text { if } c_{k} \text { is an integer; } \\
J_{k}\left[\begin{array}{c}
z^{2 c k} E_{k, 1}(z) \\
z^{2 c_{k} 0} E_{k, 0}(z)
\end{array}\right] \\
\text { if } c_{k} \text { is an odd multiple of }(1 / 2) .
\end{array}\right.
$$

Having found the necessary form for $\boldsymbol{E}(z)$, we proceed to examine which case (out of 16 possible ones) could yield nontrivial filters under the perfect-reconstruction constraint. In Table I we shall eventually summarize these 16 possibilities.

Suppose that both filters $H_{k}(z)$ are antisymmetric (i.e., $J_{0}=J_{1}=-1$ ), then they both have a zero at $z=1$. Consequently, perfect reconstruction is not possible here since we cannot reconstruct the zero-frequency component of the signal. The 12 remaining cases can be reduced further by observing that the second column in Table I is the same as its third column if we interchange the filters. Thus, there are only 8 cases to consider, namely, the cases that lie in the first and second columns of Table I.

A. $c_{0}$ and $c_{1}$ are Integers $\left(N_{0}-1\right.$ and $N_{1}-1$ are Even)

Using (7), $\tilde{E}(z)$ takes the form

$$
\begin{aligned}
\tilde{E}(z) & \triangleq\left[\begin{array}{ll}
\tilde{E}_{0,0}(z) & \tilde{E}_{1,0}(z) \\
\tilde{E}_{0,1}(z) & \tilde{E}_{1,1}(z)
\end{array}\right] \\
& =\left[\begin{array}{ll}
z^{2 c 00} E_{0,0}(z) & z^{2 c 10} E_{1,0}(z) \\
z^{2 c 01} E_{0,1}(z) & z^{2 c 11} E_{1,1}(z)
\end{array}\right]\left[\begin{array}{ll}
J_{0} & 0 \\
0 & J_{1}
\end{array}\right] .
\end{aligned}
$$

It is clear from (8) that each element $E_{k . j}(z)$ is either a symmetric or antisymmetric polynomial. Taking the determinant of both sides of (8) and noting that det $\boldsymbol{E}(z)=$ 
TABLE 1

Slmmary of All Cases For the 2-Channel PR LP FIR QMF Bank. S, A, E. and O Stand For SYMmetric, ANTISYMMETRIC, Even, and Odd, Respectively. Here, "Yes" Denotes the Existence of Nontrivial Filter Banks. "No" Denotes Nonfistence of Any Filter Bank and "Trivial" Denotes Existence of Only Trivial Filter Banks

FILTER SYMMETRY

\begin{tabular}{|l|c||c|c|c|c|}
\hline \multirow{4}{*}{} & & SS & SA & AS & AA \\
\cline { 2 - 6 } & EE & Yes & No & No & No \\
\cline { 2 - 6 } ORDER & Trivial & Trivial & Trivial & No \\
\cline { 2 - 6 } & OE & Trivial & Trivial & Trivial & No \\
\cline { 2 - 6 } & OO & No & Yes & Yes & No \\
\cline { 2 - 6 } & & & &
\end{tabular}

$\operatorname{det} \tilde{E}(z)$, we obtain

$$
\begin{aligned}
\widetilde{\operatorname{det} \boldsymbol{E}}(z)=\operatorname{det} \tilde{\boldsymbol{E}}(z)= & J_{0} J_{1}\left[z^{2(c 00)+c(1)} E_{0,0}(z) E_{1,1}(z)\right. \\
& \left.-z^{2(c 01+c(1))} E_{0.1}(z) E_{1,0}(z)\right] \\
= & J_{0} J_{1} z^{\left(c_{0}+c_{1}-1\right)} \operatorname{det} \boldsymbol{E}(z) .
\end{aligned}
$$

Since $\operatorname{det} \boldsymbol{E}(z)$ is required to be a delay (i.e., $z^{-r}$ where $r$ is a nonnegative integer), the above equation yields

$$
z^{r}=J_{0} J_{1} z^{\left.\left(c_{0}\right)+c i-1\right)} z^{-r}
$$

The only solution for $J_{0}$ and $J_{1}$ from (9) is $J_{0}=J_{1}=1$ (we have excluded the case when $J_{0}=J_{1}=-1$ above since PR is not possible here). In other words, PR is possible only if both filters are symmetric. Writing (9) in terms of the orders of $H_{k}(z)$ and using the relation $c_{k}=$ $\left(N_{k}-1\right) / 2$, we have

$$
N_{0}+N_{1}=4 r_{2}, \quad r_{2}=\text { nonnegative integer. }
$$

Due to the symmetry property of det $\boldsymbol{E}(z)$, the number of distinct constraints obtained from $\operatorname{det} \boldsymbol{E}(z)=z^{-r}$ is $\left(N_{0}\right.$ $\left.+N_{1}\right) / 4$, whereas the number of coefficients from $H_{k}(z)$ are $\left(N_{0}+N_{1}\right) / 2+1$. Hence, the number of degrees of freedom left over for the design in this case (SSEE) is $\left(N_{0}\right.$ $\left.+N_{1}\right) / 4+1$.

B. $c_{0}$ and $c_{1}$ are Odd Multiples of $(1 / 2)\left(N_{0}-1\right.$ and $N_{1}-1$ are Odd)

Using the relation in (7), $\tilde{E}(z)$ has the following form:

$$
\tilde{\boldsymbol{E}}(z)=\left[\begin{array}{cc}
z^{2 c 01} E_{0,1}(z) & z^{2 c 11} E_{1,1}(z) \\
z^{2(000} E_{0,0}(z) & z^{2 c 11} E_{1,0}(z)
\end{array}\right]\left[\begin{array}{cc}
J_{0} & 0 \\
0 & J_{1}
\end{array}\right] \text {. }
$$

Moreover, we note from (6a) and (6b) that $c_{k 0}=c_{k 1}$; thus, (11) yields

$\overparen{\operatorname{det} \boldsymbol{E}}(z)=\operatorname{det} \tilde{\boldsymbol{E}}(z)=-J_{0} J_{1} z^{2\left(\cos +c_{10}\right)} \operatorname{det} \boldsymbol{E}(z)$.

With (6a), (6b) and the PR requirement $\operatorname{det} E(z)=z^{-r}$, (12) yields

$$
z^{r}=-J_{0} J_{1} z^{c_{0}+c_{1}-1} z^{-r} .
$$

This is possible if and only if $J_{0}$ and $J_{1}$ have opposite signs, i.e., $H_{0}(z)$ and $H_{1}(z)$ have opposite symmetry. We furthermore know that $c_{00}=\left(N_{0}-2\right) / 4$, and $c_{10}=\left(N_{1}\right.$ $-2) / 4$, therefore, (13) yields

$$
N_{0}+N_{1}=4 r_{1}, \quad r_{1}=\text { nonnegative integer. }
$$

Similar to the above case, the degree of freedom in this case (SAOO) is $\left(N_{0}+N_{1}\right) / 4$.

C. $c_{0}$ and $c_{1}$ are Integer and Odd Multiple of $(1 / 2)\left(N_{0}\right.$ - 1 and $N_{1}-1$ are Even and Odd)

We will not discuss the other case where $c_{0}$ is an odd multiple of (1/2) and $c_{1}$ is an integer here since it can be similarly derived. Using (7), $\tilde{E}(z)$ takes the form

$$
\tilde{\boldsymbol{E}}(z)=\left[\begin{array}{ll}
z^{2000} E_{0,0}(z) & z^{2 c 11} E_{1,1}(z) \\
z^{2 c 01} E_{0,1}(z) & z^{2 c 10} E_{1,0}(z)
\end{array}\right]\left[\begin{array}{cc}
J_{0} & 0 \\
0 & J_{1}
\end{array}\right] .
$$

Using the fact that $c_{10}=c_{11}$ [derived from (6a) and (6b)], the determinant of $\tilde{\boldsymbol{E}}(z)$ in (15) is

$$
\begin{aligned}
\operatorname{det} \tilde{\boldsymbol{E}}(z)= & J_{0} J_{1} z^{2 c 10}\left[z^{2000} E_{0,0}(z) E_{1,0}(z)\right. \\
& \left.-z^{2 c 01} E_{0,1}(z) E_{1,1}(z)\right] .
\end{aligned}
$$

Since the degrees of $\left(z^{2200} E_{0,0}(z) E_{1,0}(z)\right)$ and $\left(z^{2 c 01}\right.$ $\left.E_{0,1}(z) E_{1,1}(z)\right)$ differ in general by one, therefore, $\operatorname{det} \boldsymbol{E}(z)$ is not naturally symmetric. Observing also that

$$
\left\{\begin{array}{l}
c_{00}=\left(N_{0}-1\right) / 4, \\
c_{01}=\left(N_{0}-3\right) / 4, \\
c_{10}=\left(N_{1}-2\right) / 4, \\
c_{11}=\left(N_{1}-2\right) / 4,
\end{array}\right.
$$

then (16) yields $\left(N_{0}+N_{1}-1\right) / 2$ constraints when we equate it with $b z^{r}$. Meanwhile, the number of coefficients are $\left(N_{0}+N_{1}+1\right) / 2$. The degree of freedom is therefore only 1 and, thus, the resulting filters are trivial. One example of such trivial PR LP pair is

$$
H_{0}(z)=\sum_{l=0}^{N_{0}-1} z^{-l}, \quad H_{1}(z)=1+z^{-1} .
$$

We summarize all the cases in Table I. From the table, we observe that there are only two distinct cases which yield nontrivial filters. These are the SAOO and SSEE cases, which we shall hereafter call Type A and Type B, respectively.

Let us now discuss the properties of the synthesis filters of a PR system. From Fig. 2, with $\boldsymbol{R}(z)=\boldsymbol{E}^{-1}(z)$, we have

$$
\left[\begin{array}{l}
F_{0}(z) \\
F_{1}(z)
\end{array}\right]=\left[\boldsymbol{E}^{-1}\left(z^{2}\right)\right]^{T}\left[\begin{array}{l}
z^{-1} \\
1
\end{array}\right] .
$$

Since $\operatorname{det} \boldsymbol{E}(z)=b z^{-r}$, the above equation becomes

$$
\left[\begin{array}{l}
F_{0}(z) \\
F_{1}(z)
\end{array}\right]=\frac{1}{b z^{-2 r}}\left[\begin{array}{rr}
0 & -1 \\
1 & 0
\end{array}\right] \boldsymbol{E}\left(z^{2}\right)\left[\begin{array}{c}
1 \\
-z^{-1}
\end{array}\right] .
$$

Simplifying the above expression, we have

$$
\left[\begin{array}{l}
F_{0}(z) \\
F_{1}(z)
\end{array}\right]=\frac{z^{2 r}}{b}\left[\begin{array}{r}
-H_{1}(-z) \\
H_{0}(-z)
\end{array}\right] .
$$


Note that the above relation (20) between the synthesis and analysis filters holds regardless of phase responses of $H_{k}(z)$. Moreover, if the analysis filters have linear phase, then so do the synthesis filters. The noncausal factor $z^{2 r}$ can be dropped in practice.

\section{Analysis Filters and Lattice Structures for TYPE A SYSTEMS}

Recall that "Type A'" implies that $\left[H_{0}(z), H_{1}(z)\right]$ is a linear-phase FIR SAOO pair, and that $\operatorname{det} \boldsymbol{E}(z)$ is a delay. Our aim here is to obtain FIR lattice structures for the pair $\left[H_{0}(z), H_{1}(z)\right]$ such that it is guaranteed to be Type A. We shall first propose a structure, and then prove that (almost) any Type A pair can be realized by the structure, so that the structure is a general tool for optimal design of such pairs of filters. (The parenthetical adjective "almost" is elaborated toward the end of this section.) The basic ingredient of the proof will be to show that such a pair can be systematically synthesized in the proposed structural form. In the following discussions, the synthesis procedures are primarily tools for such proofs.

Without loss of generality, we assume that $H_{0}(z)$ and $H_{1}(z)$ are symmetric and antisymmetric, respectively. Their lengths, $N_{0}$ and $N_{1}$, are both even. We start with a structure closely related to the well-known linear prediction lattice [8], [12] shown in Fig. 5 (for the moment ignore the last section $\left.\left[\begin{array}{ll}1 & 1 \\ 1 & -1\end{array}\right]\right)$. Traditionally, in the linear prediction lattice, the coefficients $k_{m}$, which are real valued, are constrained to be $k_{m}^{2}<1$. This constraint is a necessary and sufficient condition for the FIR transfer function $T_{N}(z)$ to have minimum-phase (i.e., all $N$ zeros strictly are inside the unit circle). The FIR function $U_{N}(z)$ is automatically constrained by the structure to be such that $U_{N}(z)=z^{-N} T_{N}\left(z^{-1}\right)=\hat{T}_{N}(z)$. Thus, $\left[T_{N}(z)\right.$, $\left.U_{N}(z)\right]$ is an MIP, and $U_{N}(z)$ is a maximum-phase FIR filter.

If we permit $k_{m}$ to be arbitrary real numbers (i.e., not constrained to be $\left.k_{m}^{2}<1\right)$, then $U_{N}(z)$ and $T_{N}(z)$ are still related as $U_{N}(z)=\hat{T}_{N}(z)$, but $U_{N}(z)$ can now have arbitrary phase response. Caution should, however, be exercised concerning the possibility of $k_{m}^{2}=1$, which leads to "singularities" as elaborated a few paragraphs later. We shall now exploit the fundamental features of this structure (unconstrained $k_{m}$, with $k_{m}^{2} \neq 1$ ) to eventually obtain Type A pairs.

Given the pair $\left[H_{0}(z), H_{1}(z)\right]$, the idea is to generate the $\operatorname{MIP}\left[T_{N}(z), U_{N}(z)\right]$, which can be synthesized in the form of a lattice. If $H_{0}(z)$ and $H_{1}(z)$ have the same lengths $\left(N_{0}=N_{1}\right)$ the MIP can be generated as

$$
\left[\begin{array}{l}
T_{N}(z) \\
U_{N}(z)
\end{array}\right]=\frac{1}{2}\left[\begin{array}{rr}
1 & 1 \\
1 & -1
\end{array}\right]\left[\begin{array}{l}
H_{0}(z) \\
H_{1}(z)
\end{array}\right] .
$$

If, on the other hand, we have $N_{0}<N_{1}$, the centers of symmetries of $H_{0}(z)$ and $H_{1}(z)$ should first be aligned. The MIP is then generated as

$$
\left[\begin{array}{l}
T_{N}(z) \\
U_{N}(z)
\end{array}\right]=\frac{1}{2}\left[\begin{array}{rr}
1 & 1 \\
1 & -1
\end{array}\right]\left[\begin{array}{l}
z^{-\left(\left(N_{i}-N_{0}\right) / 2\right)} H_{0}(z) \\
H_{1}(z)
\end{array}\right] .
$$

The inverse relation is evidently

$$
\left[\begin{array}{l}
z^{-\left(\left(N_{1}-N_{0}\right) / 2\right)} H_{0}(z) \\
H_{1}(z)
\end{array}\right]=\left[\begin{array}{rr}
1 & 1 \\
1 & -1
\end{array}\right]\left[\begin{array}{l}
T_{N}(z) \\
U_{N}(z)
\end{array}\right]
$$

which is represented in Fig. 5. The purpose of the delay $z^{-\left(N_{1}-N_{0}\right) / 2}$ is to align the centers of symmetry of both $H_{0}(z)$ and $H_{1}(z)$. For arbitrary choices of $H_{0}(z)$ and $H_{1}(z)$, the filters $T_{N}(z)$ and $U_{N}(z)$ obtained from (22) no longer have minimum phase and maximum phase. How ever, by permitting unconstrained real values for $k_{m}$, one can still realize the pair $\left[T_{N}(z), U_{N}(z)\right]$ by using the structure of Fig. 5.

To comprehend the nature of the synthesis problem at hand, we shall, for a moment, relax the perfect-reconstruction constraint. Thus, let $\left[T_{N}(z), U_{N}(z)\right]$ be any MIP of $\operatorname{order} N$. The procedure to synthesize a lattice of the form in Fig. 5 can be understood by referring to Fig. $6(a)$, which shows the $m$ th section of Fig. 5. Since the polynomials $T_{m}(z)$ and $U_{m}(z)$ are given as

$$
\left[\begin{array}{c}
T_{m}(z) \\
U_{m}(z)
\end{array}\right]=\left[\begin{array}{cc}
1 & k_{m} \\
k_{m} & 1
\end{array}\right]\left[\begin{array}{l}
T_{m-1}(z) \\
z^{-1} U_{m-1}(z)
\end{array}\right],
$$

we can invert the relation to obtain

$$
\left[\begin{array}{l}
T_{m-1}(z) \\
z^{-1} U_{m-1}(z)
\end{array}\right]=\frac{1}{1-k_{m}^{2}}\left[\begin{array}{cc}
1 & -k_{m} \\
-k_{m} & 1
\end{array}\right]\left[\begin{array}{c}
T_{m}(z) \\
U_{m}(z)
\end{array}\right],
$$

provided that $k_{m}^{2} \neq 1$. Thus, given the MIP $\left[T_{N}(z)\right.$, $\left.U_{N}(z)\right]$, we can iteratively compute the lower order pairs [ $\left.T_{k}(z), U_{k}(z)\right], k=N-1, N-2, \cdots$, by repeated application of (24), resulting in the structure of Fig. 5. The quantity $k_{m}$ which results in a reduced-order pair $\left[T_{m-1}(z), U_{m-1}(z)\right]$ is given by $k_{m}=t_{m . m} / t_{m, 0}$ where $t_{m, i}$ is the $i$ th coefficient of the impulse response of $T_{m}(z)$, i.e., $T_{m}(z)=\Sigma_{i=0}^{m} t_{m, i} z^{-i}$. It can be verified that $\left[T_{m-1}(z), U_{m-1}(z)\right]$ is an MIP (assuming, of course, that $\left[T_{m}(z), U_{m}(z)\right]$ is an MIP). This procedure is the familiar "downward recursion" in the Levinson's algorithm [8], [12], [13] with the exception that $k_{m}$ is now arbitrary (rather than constrained to be $k_{m}^{2}<1$ as in Levinson's algorithm).

Now, the above procedure works for any MIP $\left[T_{N}(z)\right.$, $\left.U_{N}(z)\right]$ provided that $k_{m}^{2} \neq 1$ at any stage. If $k_{m}^{2}=1$ for some $m$, the $2 \times 2$ matrices in (23) and (24) are singular. The meaning of this singularity situation is discussed further in Appendix A. In what follows, we shall outline a method to overcome singularity situations.

The iteration in (23) is not the only means of constructing a lower order MIP $\left[T_{m-1}(z), U_{m-1}(z)\right]$ from an MIP $\left[T_{m}(z), U_{m}(z)\right]$. A more general procedure would be to define

$$
T_{m-1}(z)=\frac{1}{1-k_{m}^{2}}\left(\frac{1}{1-z^{-1} z_{m}}\right)\left[T_{m}(z)-k_{m} U_{m}(z)\right]
$$




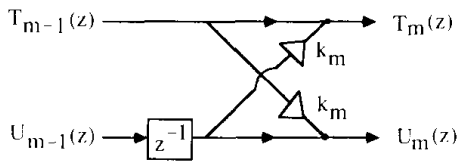

(a)

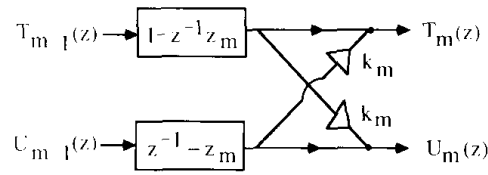

(b)

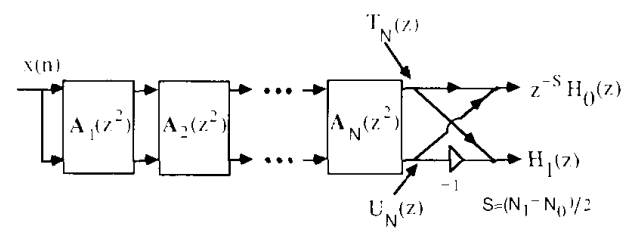

(c)

Fig. 6. (a) Type I building block. (b) Type II building block for the SAOO or SAEE LP FIR lattice structure. (c) The SAOO or SAEE LP FIR lattice structure.

and

$$
U_{m-1}(z)=\frac{1}{1-k_{m}^{2}}\left(\frac{1}{z^{-1}-z_{m}}\right)\left[-k_{m} T_{m}(z)+U_{m}(z)\right]
$$

which works as follows: $z_{m}$ is an arbitrary real number. The parameter $k_{m}$ is chosen to be

$$
k_{m}=\frac{T_{m}\left(z_{m}\right)}{U_{m}\left(z_{m}\right)}
$$

so that $T_{m}(z)-k_{m} U_{m}(z)$ has a zero at $z=z_{m}$, cancelling the denominator $1-z^{-1} z_{m}$ in (25a). Since $\left[T_{m}(z)\right.$, $\left.U_{m}(z)\right]$ is MIP, the factor $\left(z^{-1}-z_{m}\right)$ is cancelled off in (25b) by the same choice of $k_{m}$. Furthermore, since the pair $\left[1-z^{-1} z_{m}, z^{-1}-z_{m}\right]$ is MIP, the pair $\left[T_{m-1}(z)\right.$, $\left.U_{m-1}(z)\right]$ is guaranteed to be a reduced-order FIR MIP!

The purpose of the number $z_{m}$ is to avoid the possibility of $k_{m}^{2}$ being unity. Since $T_{m}(z) \pm U_{m}(z)$ is a finite degree polynomial, it has only $m$ zeros so that there is guaranteed to exist $z_{m}$ such that $T_{m}\left(z_{m}\right)+U_{m}\left(z_{m}\right) \neq 0, T_{m}\left(z_{m}\right)-$ $U_{m}\left(z_{m}\right) \neq 0$, and $U_{m}\left(z_{m}\right) \neq 0$. Except for this restriction, $z_{m}$ is entirely arbitrary. In order to find a $z_{m}$ that works, it is only necessary to try out at most $3 m$ values, say, $z_{m}=2^{-k}, k=0,1, \cdots, 3 m-1$. In Appendix B, it is shown that such a $z_{m}$ is guaranteed to exist as long as there is no common factor between $T_{N}(z)$ and $U_{N}(z)$. (It is also shown in Appendix $C$ that such a common factor cannot exist if $\left[H_{0}(z), H_{1}(z)\right]$ is a PR pair.) Fig. 6(b) shows the structural interpretation of the new order-reduction scheme. The resulting structure for arbitrary MIP $\left[T_{N}(z), U_{N}(z)\right]$ is as in Fig. 6(c) with building blocks $\boldsymbol{A}_{m}(z)$ as in Fig. 6(b). It is clear that any arbitrary MIP $\left[T_{N}(z), U_{N}(z)\right]$ can be represented in this form, and that for arbitrary $k_{m}, z_{m}\left(k_{m}^{2} \neq 1\right)$, the structure gives rise to only MIP $\left[T_{N}(z), U_{N}(z)\right]$. Notice that the traditional structure of Fig. 5 is obtained as a special case with $z_{m}=$ 0 . Readers familiar with the relation between the linearprediction IIR lattice and FIR lattice structure [8] will recognize that the relation between Fig. 6(b) and the IIR allpass structures in $[13$, p. 483$]$ is similar. For convenience of discussion, the building blocks in Fig. 6(a) and (b) will, respectively, be called Types I and II. These are equivalent when $z_{m}=0$.

The structures for $\left[H_{0}(z), H_{1}(z)\right]$ proposed in Figs. 57 are not in the form of Fig. 4, and this gap should now be bridged. First consider Fig. 5. It is clear that we can represent $\left[H_{0}(z), H_{1}(z)\right]$ as

$$
\left[\begin{array}{l}
H_{0}(z) \\
H_{1}(z)
\end{array}\right]=G(z)\left[\begin{array}{c}
1 \\
z^{-1}
\end{array}\right]
$$

where $\boldsymbol{G}(z)$ is a $2 \times 2$ FIR transfer matrix with $\operatorname{det} \boldsymbol{G}(z)$ $=$ delay. However, since this is not in the form (3) [i.e., $\boldsymbol{G}(z)$ is not equal to $\boldsymbol{E}\left(z^{2}\right)$ ], we cannot conclude that $\operatorname{det} \boldsymbol{E}(z)=$ delay. Thus, it is not true that all $\left[H_{0}(z)\right.$, $H_{1}(z)$ ] pairs represented by Fig. 5 lead to FIR perfectreconstruction analysis banks. If we impose the stipulation that $k_{m}=0$ for even $m$ in Fig. 5, then $\boldsymbol{G}(z)=\boldsymbol{E}\left(z^{2}\right)$, and det $\boldsymbol{E}(z)$ is indeed a delay. What is more important, however, is the following stronger result.

Lemma 3.1: Let $\left[H_{0}(z), H_{1}(z)\right]$ be a Type A pair such that we can synthesize it in the form of Fig. 5, such that $k_{m} \neq \pm 1$ for any $m$. Then the even-numbered coefficients $k_{2 m}$ automatically turn out to be zero, if $\operatorname{det} \boldsymbol{E}(z)$ is a delay.

Proof: The proof is inductive. Suppose that we are given an $m$ th-order $\operatorname{MIP}\left[T_{m}(z), U_{m}(z)\right]$ which at the same time is a $\mathrm{PR}$ pair, i.e.,

$$
\left[\begin{array}{c}
T_{m}(z) \\
U_{m}(z)
\end{array}\right]=\boldsymbol{E}_{m}\left(z^{2}\right)\left[\begin{array}{c}
1 \\
z^{-1}
\end{array}\right], \quad \text { with } \operatorname{det} \boldsymbol{E}_{m}(z)=c z^{-r} .
$$

The coefficient of the $z^{0}$ power in $\operatorname{det} \boldsymbol{E}_{m}(z)$ is $\left(t_{m, 0} t_{m, m-1}-t_{m, 1} t_{m, m}\right)$ which should be 0 . This condition, together with $k_{m}=t_{m, m} / t_{m, 0}$, yields

$$
k_{m}=\frac{t_{m, m}}{t_{m, 0}}=\frac{t_{m, m-1}}{t_{m, 1}} .
$$

Substituting (28b) in (24), we obtain $t_{m-1 . m-1}=u_{m-1.0}$ $=0$. Thus, an order reduction by two is automatically enforced, so that

$$
\left[\begin{array}{l}
T_{m-2}(z) \\
z^{-2} U_{m-2}(z)
\end{array}\right]=\frac{1}{\left(1-k_{m}^{2}\right)}\left[\begin{array}{rr}
1 & -k_{m} \\
-k_{m} & 1
\end{array}\right]\left[\begin{array}{c}
T_{m}(z) \\
U_{m}(z)
\end{array}\right] .
$$

By combiñing (29) and (28a), we also see that $\left[T_{m-2}(z)\right.$, $\left.U_{m-2}(z)\right]$ is an MIP PR pair. So if we start with an MIP PR pair $\left[T_{N}(z), U_{N}(z)\right]$ and repeat the above order re- 


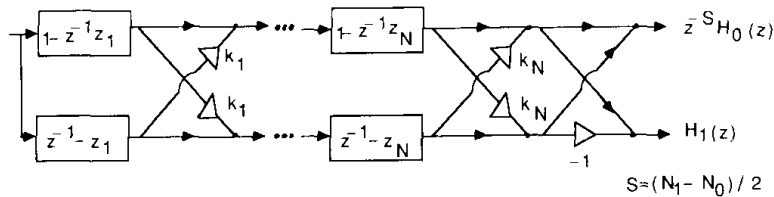

Fig. 7. The SAOO or SAEE LP FIR lattice structure.

duction process, we see that $k_{m}=0$ for even $m$ and Fig. 8 reduces to a PR structure as shown in Fig. 9. QED

Since we restrict $z_{m}$ to be zero in Lemma 3.1, it therefore does not cover all Type A pairs of filters. The pairs of filters which the structure of Fig. 9 excludes are essentially the ones which, during the synthesis process, yield internal transfer function pairs (the $\left[T_{m}(z), U_{m}(z)\right]$ pairs) such that $t_{m, 0}= \pm t_{m, m}$ forcing $k_{m}= \pm 1$. Suppose that the optimal filter happens to be one of these excluded filters. Then, in practice, we can still always get as close to it as possible by using the structure of Fig. 9. Thus, these excluded filters are not expected to result in serious loss of generality.

A result similar to Lemma 3.1 does not hold for the structure with the generalized building blocks shown in Fig. 7. Even if such a result were true, the quantity $\boldsymbol{E}^{-1}(z)$ which arises in the synthesis bank would contain the inverses of these building blocks. These inverse building blocks would contain the two factors $1 /\left(1-z^{-1} z_{m}\right)$ and $1 /\left(z^{-1}-z_{m}\right)$ at least one of which is necessarily unstable (i.e., synthesis filters are IIR and unstable) for any choice of $z_{m}$. This observation excludes the consideration of Fig. 7 for the rest of this paper.

Example 3.1: In the optimization procedure, we additionally use two scale factors $\beta_{1}$ and $\beta_{2}$ at the end of the structure in Fig. 9. The objective function to be minimized is

$$
\begin{aligned}
\Phi= & \int_{0}^{\omega_{p}}\left[1-\left|H_{0}\left(e^{j \omega}\right)\right|\right]^{2} d \omega+\int_{\omega S}^{\pi}\left[H_{0}\left(e^{j \omega}\right)\right]^{2} d \omega \\
& +\int_{\omega S}^{\pi}\left[1-\left|H_{1}\left(e^{j \omega}\right)\right|\right]^{2} d \omega+\int_{0}^{\omega_{p} p}\left[H_{1}\left(e^{j \omega}\right)\right]^{2} d \omega .
\end{aligned}
$$

A mathematical optimization subroutine [15] is used to search for an optimal solution. In order to initialize the lattice coefficients, we use the tabulated linear-phase filters designed earlier by Johnston [22], [1, p. 401]. For our example, the filter 64D was used for $H_{0}(z)$, and $H_{1}(z)$ was taken to be $H_{0}(-z)$. With this "initial pair," the lattice of Fig. 5 was synthesized. Since the filters obtained from [1, p. 401] give only an approximation to a $P R$ pair, the even-numbered coefficients $k_{2 m}$ do not turn out to be zero. These were forced to be zero during initialization, and the odd-numbered coefficients reoptimized using [15]. The frequency responses of these reoptimized filters are shown in Fig. 10(a). The number of lattice sections in the example is equal to 32 and the transition bandwidth is $\Delta f$

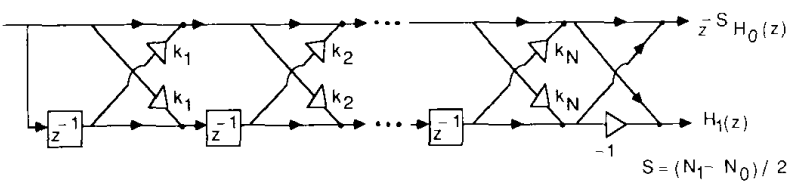

Fig. 8. A redrawing of Fig. 7 with $z_{m}=0$.

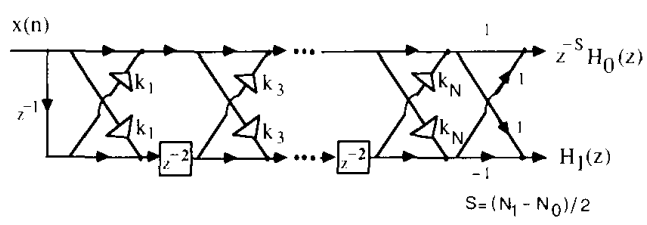

Fig. 9. The lattice structure for Type A system. $N$ is odd.

$=0.086$. Notice that these filters form an exact PR pair, and retain the $\mathrm{PR}$ property in spite of quantization of the coefficients $k_{m}$ to any desired level. The synthesis filters $F_{0}(z)$ and $F_{1}(z)$ can be obrained by using (20). Table II displays the lattice coefficients $k_{2 m+1}$, and the impulse responses of both analysis filters. Table II displays only the first half of the coefficients of $H_{0}(z)$ and $H_{1}(z)$ since the impulse responses are symmetric and antisymmetric, respectively.

It is interesting to compare the above perfect-reconstruction design with Johnston's 64D filter in [1, p. 401] (to be referred to as the 64D filter in the following discussion). Both designs have linear phase and filter lengths equal to 64 . The PR pair has a minimum stopband attenuation of about $42 \mathrm{~dB}$, and reconstruction error equal to zero. On the other hand, the $64 \mathrm{D}$ filter has a better minimum stopband attenuation of about $65 \mathrm{~dB}$, but a nonzero reconstruction error (defined in [1]) of about $0.002 \mathrm{~dB}$. Both designs have about the same transition bandwidth, viz., $\Delta f=0.086$. The tradeoff is therefore very clear. For comparison, the frequency responses of the 64D-analysis filters are shown in Fig. 10(b), and the impulse response coefficients in Table III. Notice that the significantly large coefficients in the 64D filter agree closely with those in the PR pair. The 64D filters satisfy the relation $H_{1}(z)=$ $H_{0}(-z)$, whereas the PR pair does not. It can in fact be shown that, with $H_{1}(z)=H_{0}(-z)$, it is impossible to force det $\boldsymbol{E}(z)$ to be an exact delay unless $H_{0}(z)$ is a trivial function (see Appendix D). Notice, finally, that some of the trailing coefficients in the PR pair (Table II) are very small (compared to the ones in Table III). These can be replaced with "zero"' without significant effect on the PR property.

A second major difference between the above PR design and the $64 \mathrm{D}$ filter is that the 64D pair $\left[H_{0}(z), H_{1}(z)\right]$ is approximately power complementary, i.e., $\left|H_{0}\left(e^{j \omega}\right)\right|^{2}$ $+\left|H_{1}\left(e^{j \omega}\right)\right|^{2}=1$ [see Fig. 10(c)]. In fact, the design procedure in $[22]$ is such that $\int_{0}^{\pi}\left[1-\left|H_{0}\left(e^{j \omega}\right)\right|^{2}-\right.$ $\left.\left|H_{1}\left(e^{j \omega}\right)\right|^{2}\right]^{2} d \omega$ is minimized. On the other hand, the PR pair does not satisfy the power-complementary property [see Fig. 10(c)]. In fact, the main basis of the PR designs 


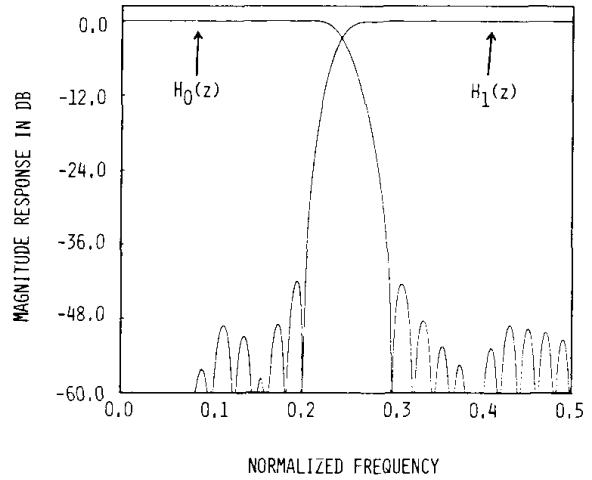

(a)

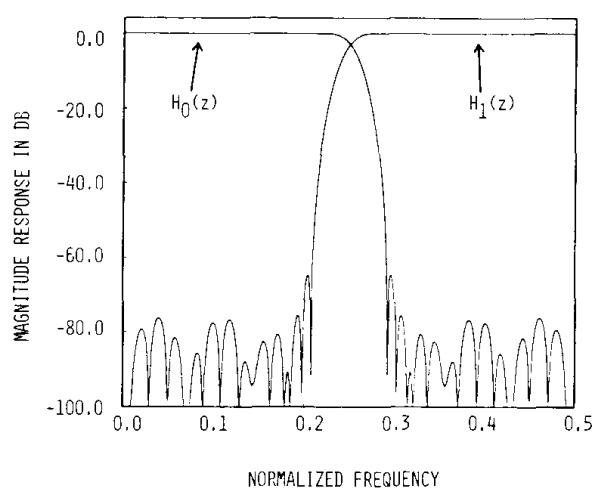

(b)

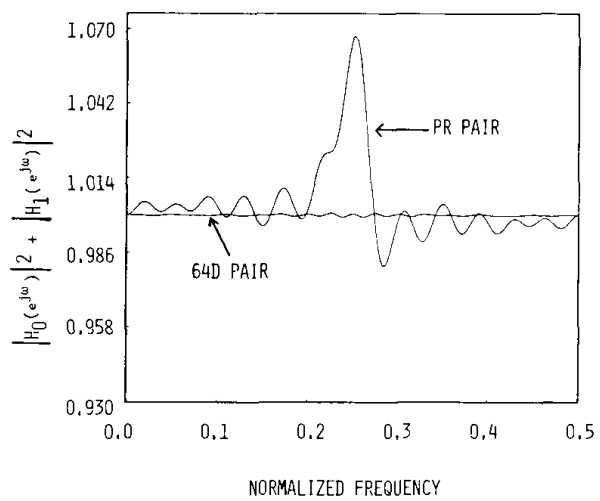

(c)

Fig. 10. (a) Example 3.1: Magnitude response plots for the optimized analysis filters. Each filter has length $=64$. (b) Example 3.1: Magnitude response plots for the $64 \mathrm{D}$ analysis filters. Each filter has length $=64$. (c) Example 3.1: The plots of $\left|H_{0}\left(e^{j \omega}\right)\right|^{2}+\left|H_{1}\left(e^{j \omega}\right)\right|^{2}$ for the 64D pair of filters and the new $P R$ pair of filters.

introduced in this paper is the fact that PR property can be accomplished exactly for linear-phase filters, by giving up power complementarity of the pair $\left[H_{0}(z), H_{1}(z)\right]$ as well as the relation $H_{1}(z)=H_{0}(-z)$ which are two restrictions forced in most of the earlier designs [16], [22][24]. After relaxing these two restrictions, we can obtain good PR pairs which in addition have linear phase.

Implementation Complexity: A further interesting point of comparison between the 64D design and our PR design
TABLE II

Lattice Coefficients and Impulse Responses of the Optimized ANALYSIS Filters IN EXAMPLE 3.1. (SAOO) $\beta_{1}=9.3367072622762 \times$ $10^{-10}, \beta_{2}=8.6458769493813 \times 10^{-10}$

\begin{tabular}{|c|c|c|c|}
\hline$m$ & Lattice Coeff. $k_{2 m+1}$ & Filter Coefficients $h_{0, m}$ & Filter Coefficients $h_{1, m}$ \\
\hline 0 & -0.16748024178056 & $-2.8047649315578 \times 10^{-8}$ & $2.7701557075405 \times 10^{-8}$ \\
\hline 1 & -0.98630142049519 & $4.6974270887493 \times 10^{-8}$ & $-4.6394634766867 \times 10^{-8}$ \\
\hline 2 & 46.797422738757 & $-9.0320483808830 \times 10^{-6}$ & $8.8828615641826 \times 10^{-6}$ \\
\hline 3 & 1.0155415002447 & $1.5395771337727 \times 10^{-6}$ & $-1.5142595131503 \times 10^{-6}$ \\
\hline 4 & -0.98123943672420 & $-3.8484265421346 \times 10^{-4}$ & $3.6797322378863 \times 10^{-4}$ \\
\hline 5 & 71.799272118326 & $7.3072834050915 \times 10^{-5}$ & $-7.0105005042002 \times 10^{-6}$ \\
\hline 6 & 0.99604836163496 & $-5.4844133513744 \times 10^{-4}$ & $3.3979745779936 \times 10^{-6}$ \\
\hline 7 & 73.293136853215 & $4.4822758623806 \times 10^{-4}$ & $-3.4610132423983 \times 10^{-4}$ \\
\hline 8 & -0.32992582104225 & $-3.1420371203276 \times 10^{-4}$ & $-2.9314605847016 \times 10^{-6}$ \\
\hline 9 & -0.58756852009572 & $6.0057015567620 \times 10^{-5}$ & $4.6300165292477 \times 10^{-4}$ \\
\hline 10 & 0.68608642287498 & $9.0093326906975 \times 10^{-4}$ & $-9.6019269511046 \times 10^{-4}$ \\
\hline 11 & -6.8758613928422 & $1.6949632352623 \times 10^{-4}$ & $-5.3867082067374 \times 10^{-4}$ \\
\hline 12 & -1.0899663381059 & $-1.7113555648237 \times 10^{-3}$ & $2.6665204377661 \times 10^{-5}$ \\
\hline 13 & 0.70138304837561 & $-8.85866614964934 \times 10^{-4}$ & $1.3763566255439 \times 10^{-3}$ \\
\hline 14 & 1.9359402130086 & $2.1821453210951 \times 10^{-3}$ & $-3.9203746653654 \times 10^{-3}$ \\
\hline 15 & 32.412571811713 & $1.8133626090971 \times 10^{-3}$ & $-3.2204697559136 \times 10^{-3}$ \\
\hline 16 & -2.2762581355052 & $-2.5292916536995 \times 10^{-3}$ & $5.2062396452593 \times 10^{-5}$ \\
\hline 17 & -1.0546765432115 & $-4.1596794940782 \times 10^{-3}$ & $6.1298978924939 \times 10^{-5}$ \\
\hline 18 & -14.637818055814 & $3.5887675000538 \times 10^{-3}$ & $-6.2739942846780 \times 10^{-5}$ \\
\hline 19 & 0.91309339992145 & $7.6713479773340 \times 10^{-3}$ & $-1.0972495757049 \times 10^{-2}$ \\
\hline 20 & 0.20497603236147 & $-5.7266129912836 \times 10^{-3}$ & $6.2975034654359 \times 10^{-9}$ \\
\hline 21 & -8.7549576287234 & $-1.2745794143760 \times 10^{-2}$ & $1.8004225149880 \times 10^{-2}$ \\
\hline 22 & -34.634777587712 & $9.5185739377873 \times 10^{-3}$ & $-4.2379770697116 \times 10^{-3}$ \\
\hline 23 & 0.84932894911060 & $2.0342217498768 \times 10^{-2}$ & $-2.7168914395689 \times 10^{-2}$ \\
\hline 24 & -2.7082137206053 & $-1.6072095730447 \times 10^{-2}$ & $-1.0169910794786 \times 10^{-9}$ \\
\hline 25 & -1.0358588647791 & $-3.1588256584766 \times 10^{-3}$ & $3.9969783616942 \times 10^{-2}$ \\
\hline 26 & 11.636158464610 & $2.7807655367843 \times 10^{-2}$ & $1.2025400363970 \times 10^{-2}$ \\
\hline 27 & 1.2034512561861 & $5.0150998969974 \times 10^{-2}$ & $-6.0718905144588 \times 10^{-2}$ \\
\hline 28 & -0.20699149394524 & $-5.2720604380285 \times 10^{-2}$ & $-3.6911412178463 \times 10^{-2}$ \\
\hline 29 & -0.19132416529613 & $-9.3506916444599 \times 10^{-2}$ & $1.0590613371084 \times 10^{-1}$ \\
\hline 30 & 0.68112439023728 & $1.4064635171445 \times 10^{-1}$ & $1.2541814981813 \times 10^{-1}$ \\
\hline 31 & -31.040193536859 & $4.5677057793319 \times 10^{-1}$ & $-4.7085951885497 \times 10^{-1}$ \\
\hline
\end{tabular}

TABLE III

IMPULSE RESPONSES OF THE JOHNSTON'S 64D FILTERS IN EXAMPLE 3.1

\begin{tabular}{|c||r|r|}
\hline \hline$m$ & Coefficients $h_{0, m}$ & \multicolumn{1}{|c}{ Coefficients $h_{1, m}$} \\
\hline 0 & $3.596189 \times 10^{-6}$ & $3.596189 \times 10^{-6}$ \\
\hline 1 & $-1.123515 \times 10^{-4}$ & $1.123515 \times 10^{-4}$ \\
\hline 2 & $-1.104587 \times 10^{-4}$ & $-1.104587 \times 10^{-4}$ \\
\hline 3 & $2.790277 \times 10^{-4}$ & $-2.790277 \times 10^{-4}$ \\
\hline 4 & $2.298438 \times 10^{-4}$ & $2.298438 \times 10^{-4}$ \\
\hline 5 & $-5.953563 \times 10^{-4}$ & $5.953563 \times 10^{-4}$ \\
\hline 6 & $-3.823631 \times 10^{-4}$ & $-3.823631 \times 10^{-4}$ \\
\hline 7 & $1.138260 \times 10^{-3}$ & $-1.138260 \times 10^{-3}$ \\
\hline 8 & $5.308539 \times 10^{-4}$ & $5.308539 \times 10^{-4}$ \\
\hline 9 & $-1.986177 \times 10^{-3}$ & $1.986177 \times 10^{-3}$ \\
\hline 10 & $-6.243724 \times 10^{-4}$ & $-6.243724 \times 10^{-4}$ \\
\hline 11 & $3.235877 \times 10^{-3}$ & $-3.235877 \times 10^{-3}$ \\
\hline 12 & $5.743159 \times 10^{-4}$ & $5.743159 \times 10^{-4}$ \\
\hline 13 & $-4.948147 \times 10^{-3}$ & $4.948147 \times 10^{-3}$ \\
\hline 14 & $-2.583767 \times 10^{-4}$ & $-2.583767 \times 10^{-4}$ \\
\hline 15 & $7.367171 \times 10^{-3}$ & $-7.367171 \times 10^{-5}$ \\
\hline 16 & $-4.857935 \times 10^{-4}$ & $-4.857935 \times 10^{-4}$ \\
\hline 17 & $-1.050689 \times 10^{-2}$ & $1.050689 \times 10^{-2}$ \\
\hline 18 & $1.894714 \times 10^{-3}$ & $1.894714 \times 10^{-3}$ \\
\hline 19 & $1.459396 \times 10^{-5}$ & $-1.459396 \times 10^{-2}$ \\
\hline 20 & $-4.313674 \times 10^{-3}$ & $-4.313674 \times 10^{-3}$ \\
\hline 21 & $-1.994365 \times 10^{-2}$ & $1.994365 \times 10^{-2}$ \\
\hline 22 & $8.287560 \times 10^{-3}$ & $8.287560 \times 10^{-3}$ \\
\hline 23 & $2.716055 \times 10^{-2}$ & $-2.716055 \times 10^{-2}$ \\
\hline 24 & $-1.485397 \times 10^{-2}$ & $-1.485397 \times 10^{-2}$ \\
\hline 25 & $-3.764973 \times 10^{-2}$ & $3.764973 \times 10^{-3}$ \\
\hline 26 & $2.644700 \times 10^{-2}$ & $2.644700 \times 10^{-2}$ \\
\hline 27 & $5.543245 \times 10^{-2}$ & $-5.543245 \times 10^{-3}$ \\
\hline 28 & $-5.095487 \times 10^{-2}$ & $-5.095487 \times 10^{-2}$ \\
\hline 29 & $-9.779096 \times 10^{-2}$ & $9.779096 \times 10^{-2}$ \\
\hline 30 & $1.382363 \times 10^{-1}$ & $1.382363 \times 10^{-1}$ \\
\hline 31 & $4.600981 \times 10^{-1}$ & $-4.600981 \times 10^{-1}$ \\
\hline & & \\
\hline 3
\end{tabular}


TABLE IV

Comparison Between Johnston's 64D Filters and the New PR FILTERS

\begin{tabular}{|c||c|c|}
\hline Feature & $\begin{array}{c}\text { Johnston's 64D } \\
\text { Pair of Filters }\end{array}$ & $\begin{array}{c}\text { The new PR } \\
\text { Pair of Filters }\end{array}$ \\
\hline Phase Response & Linear & Linear \\
\hline Filter Length & 64 & 64 \\
\hline $\begin{array}{c}\text { Stopband } \\
\text { Attenuation }\end{array}$ & $65 \mathrm{~dB}$ & $42.5 \mathrm{~dB}$ \\
\hline $\begin{array}{c}\text { Reconstruction } \\
\text { Error }\end{array}$ & $0.002 \mathrm{~dB}$ & No error \\
\hline $\begin{array}{c}\text { Number of MPU } \\
\text { for Analysis Bank } \\
\text { Implementation }\end{array}$ & 32 & 17 \\
\hline $\begin{array}{c}\text { Number of APU } \\
\text { for Analysis Bank } \\
\text { Implementation }\end{array}$ & 32 & 49 \\
\hline $\begin{array}{c}\text { Power Comple- } \\
\text { mentarity }\end{array}$ & Approximately \\
holds & $\begin{array}{c}\text { Does not } \\
\text { hold }\end{array}$ \\
\hline $\begin{array}{c}\text { Relation between } \\
\text { Analysis Filters }\end{array}$ & $H_{1}(z)=H_{0}(-z)$ & $\begin{array}{c}\text { Not explicit. } \\
\text { Implicitly } \\
\text { such that }\end{array}$ \\
\hline $\begin{array}{c}\text { Overall Group } \\
\text { Delay of } \\
\text { QMF bank }\end{array}$ & 63 & det E $(z)=$ delay \\
\hline
\end{tabular}

is the number of multiplication and addition operations per unit time (abbreviated MPU and APU, respectively) ${ }^{2}$ required to implement the analysis bank. A direct-form implementation of the 64D filter pair $\left[H_{0}(z), H_{1}(z)\right]$ would appear to require $2 \times 64=128 \mathrm{MPU}$. However, there are three simplifying factors involved, viz.: a) the relation $H_{1}(z)=H_{0}(-z)$; b) the symmetry relation $h_{0}(n)$ $=h_{0}\left(N^{\prime}-1-n\right)$ where $N^{\prime}$ is the length of $H_{0}(z)$; and c) the decimation by a factor of 2 . Only two of these three factors can be simultaneously exploited, due to the fact that $N^{\prime}$ is even (see [16] for details). As a result, we require $128 / 4=32 \mathrm{MPU}$ to implement the $64 \mathrm{D}$ pair using a direct-form polyphase structure. The number of APU's required is 32 .

Now consider the PR pair of length 64 designed above [Table II and Fig. 10(a)], implemented in lattice form of Fig. 9. There are 32 lattice sections in the structure. Each lattice section can be implemented efficiently using only one multiplication and three addition operations (see [17] and [18]). Second, both the decimators can be moved to the left of all the building blocks in Fig. 9 (which are functions of $z^{2}$ ) by replacing $z^{-2}$ with $z^{-1}$ in these building blocks (see [1] or use the identity (a) in Fig. 11 of [19]). With such rearrangement, each lattice building block operates at the lower rate, and so the total number of MPU's is equal to $(32+2) / 2=17$. The number of APU's required can be similarly verified to be 49 . In conclusion, for the same filter length, the $P R$ pair requires only $17 \mathrm{MPU}$ and $49 \mathrm{APU}$, whereas the 64D pair requires 32 MPU and 32 APU.

The significant features of the above comparison are summarized in Table IV. As a final observation, suppose

${ }^{2}$ A unit of time is defined to be the sampling period of the input sequence $x(n)$ in Fig. 1 . we consider Johnston's 32D pair in [1]. This filter has the same number of MPU and nearly the same attenuation as the PR pair under discussion, and in addition has reconstruction error $=0.025 \mathrm{~dB}[1]$. In other words, the $32 \mathrm{D}$ pair and the above PR pair have nearly the same cost (assuming that the multiplication time significantly dominates addition time) and same performance, except the reconstruction error. The only price paid for perfect reconstruction appears to be the overall group delay of the QMF bank, which is 63 for the $P R$ pair, and 31 for the 32D pair.

The structure in Fig. 9 can be partly generalized for the case where $M$ is an even integer. We briefly discuss this in Appendix E.

\section{Analysis Filters and Lattice Structures for TyPE B Systems}

Recall that for a Type B pair, the FIR filters $H_{0}(z)$ and $H_{1}(z)$ have even orders and symmetric impulse responses, and the determinant of $\boldsymbol{E}(z)$ is a delay. Let [ $\left.H_{0}(z), H_{1}(z)\right]$ be a Type B pair with orders $N_{0}-1$ and $N_{1}-1$, respectively. Without loss of generality, assume that $N_{1} \geq N_{0}$. As we have shown from Section III, i.e., (10), the lengths of these filters satisfy the condition

$$
N_{0}+N_{1}=4 r_{1}, \quad r_{1}=\text { nonnegative integer }
$$

which we call the "length condition."' Based on the fact that both $N_{0}$ and $N_{1}$ are odd, it can be shown that the only choice for $N_{0}$ and $N_{1}$ which satisfies (31) is

$$
N_{1}=N_{0}+4 L+2, \quad L=\text { nonnegative integer. }
$$

In other words, the lengths of any Type B pair of filters have to obey both (31) and (32) simultaneously.

We would like to find a structure which covers all Type B pairs of filters $\left[H_{0}(z), H_{1}(z)\right]$. We accomplish this by showing that every such pair can be synthesized as a cascaded lattice. The building blocks of the lattice will be such that the Type B property propagates down the structure. Consider Fig. 11(a) where the pair $\left[P_{r}(z), Q_{m}(z)\right]$ is a Type $\mathrm{B}$ pair with orders $r=(m-4 l-2)$ and $m$, respectively, with even $m$. Let $P_{r}(z)=\sum_{j=0}^{m-4 l-2} p_{r, j} z^{-j}$ and $Q_{m}(z)=\Sigma_{j=0}^{m} q_{m, j} z^{-j}$. We shall assume that

$$
p_{r, 0}=q_{m .0} \neq 0
$$

which will be justified soon. Since $m$ is even, the order of $\left[P_{r}(z), Q_{m}(z)\right]$ satisfies the length condition.

For convenience, let us express the pair $\left[P_{r}(z), Q_{m}(z)\right]$ in polyphase form, i.e.,

$$
\begin{aligned}
{\left[\begin{array}{l}
P_{r}(z) \\
Q_{m}(z)
\end{array}\right] } & =\left[\begin{array}{ll}
P_{r, 0}\left(z^{2}\right) & P_{r .1}(z) \\
Q_{m, 0}\left(z^{2}\right) & Q_{m .1}\left(z^{2}\right)
\end{array}\right]\left[\begin{array}{c}
1 \\
z^{-1}
\end{array}\right] \\
& =\boldsymbol{E}_{m}\left(z^{2}\right)\left[\begin{array}{c}
1 \\
z^{-1}
\end{array}\right]
\end{aligned}
$$




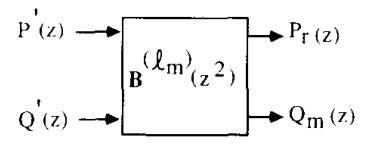

(a)

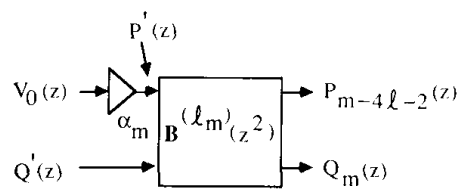

(b)

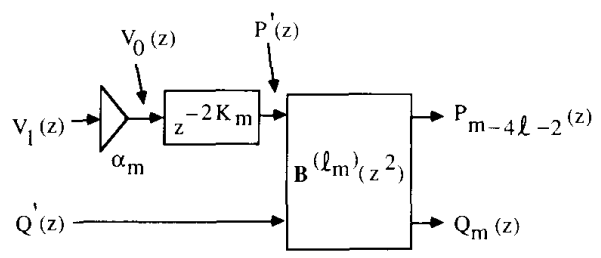

(c)

Fig. 11. (a) Pertaining to the downward recursion of Type B system. (b) The building block for Type B system where both $p_{0}^{\prime} \neq 0$ and $q_{0}^{r} \neq 0$ (c) The building block for Type B system.

The elements of $\boldsymbol{E}_{m}(z)$ have orders given by

$$
\text { Order }\left[\boldsymbol{E}_{m}(z)\right]=\left[\begin{array}{ll}
\frac{m}{2}-2 l-1 & \frac{m}{2}-2 l-2 \\
\frac{m}{2} & \frac{m}{2}-1
\end{array}\right]
$$

The determinant $P_{r, 0}(z) Q_{m, 1}(z)-P_{r, 1}(z) Q_{m, 0}(z)$ is a linear-phase polynomial (because $P_{r}(z)$ and $Q_{m}(z)$ are linear-phase polynomials of even order). Since this determinant is also equal to a delay, the following equation holds:

$$
P_{r, 0}(z) Q_{m, 1}(z)-P_{r, 1}(z) Q_{m, 0}(z)=z^{-(m / 2-1-1)} .
$$

Our aim is to use (36) to extract the building block $\boldsymbol{B}^{\left(l_{m}\right)}\left(z^{2}\right)$ in Fig. 11(a) such that the remainder pair $\left[P^{\prime}(z), Q^{\prime}(z)\right]$ is also a Type B pair with orders ( $m-$ $4 l-4)$ and $(m-4 l-2)$, respectively. Since both pairs $\left[P_{r}(z), Q_{m}(z)\right]$ and $\left[P^{\prime}(z), Q^{\prime}(z)\right]$ are PR pairs, the building block should be a function of $z^{2}$, which justifies the notation $\boldsymbol{B}^{\left(l_{m}\right)}\left(z^{2}\right)$. Furthermore, the determinant of $\boldsymbol{B}^{\left(I_{m}\right)}\left(z^{2}\right)$ is required to be a delay, in view of the PR property.

Denoting the elements of $\boldsymbol{B}^{\left(l_{m}\right)}(z)$ by $B_{i, j}^{\left(l_{m}\right)}(z)$, Fig. 11 (a) yields

$$
\begin{aligned}
{\left[\begin{array}{l}
P_{r}(z) \\
Q_{m}(z)
\end{array}\right] } & =\left[\begin{array}{ll}
B_{0.0}^{\left(l_{m}\right)}\left(z^{2}\right) & B_{0.1}^{\left(l_{m}\right)}\left(z^{2}\right) \\
B_{1,0}^{\left(l_{m}\right)}\left(z^{2}\right) & B_{1.1}^{\left(l_{m}\right)}\left(z^{2}\right)
\end{array}\right]\left[\begin{array}{l}
P^{\prime}(z) \\
Q^{\prime}(z)
\end{array}\right] \\
& =\left[\begin{array}{l}
B_{0.0}^{\left(l_{m}\right)}\left(z^{2}\right) P^{\prime}(z)+B_{0.1}^{\left(l_{m}\right)}\left(z^{2}\right) Q^{\prime}(z) \\
B_{1,0}^{\left(l_{m}\right)}\left(z^{2}\right) P^{\prime}(z)+B_{1.1}^{\left(l l_{m}\right)}\left(z^{2}\right) Q^{\prime}(z)
\end{array}\right] .
\end{aligned}
$$

The orders of the components in (37) are related as

$$
\begin{aligned}
& {\left[\begin{array}{ll}
m-4 l-2 \\
m
\end{array}\right] } \\
&=\left[\begin{array}{ll}
\operatorname{Order}\left[B_{0.0}^{\left(l_{m}\right)}\left(z^{2}\right)\right] & \text { Order }\left[B_{0.1}^{\left(l_{m}\right)}\left(z^{2}\right)\right] \\
\operatorname{Order}\left[B_{1,0}^{\left(l_{m}\right)}\left(z^{2}\right)\right] & \operatorname{Order}\left[B_{1,1}^{\left(l_{m}\right)}\left(z^{2}\right)\right]
\end{array}\right] \\
& \cdot\left[\begin{array}{l}
m-4 l-4 \\
m-4 l-2
\end{array}\right] .
\end{aligned}
$$

Both pairs $\left[P_{r}(z), Q_{m}(z)\right]$ and $\left[P^{\prime}(z), Q^{\prime}(z)\right]$ are symmetric polynomials, therefore, it can be shown that $B_{i, j}^{\left(l_{m}\right)}\left(z^{2}\right)$ also have to be symmetric polynomials. Moreover, from the first equation of (38), in order to yield a symmetric polynomial on the left-hand side, $B_{0,0}^{\left(l_{m}\right)}\left(z^{2}\right)$ $P^{\prime}(z)$ and $B_{0,1}^{\left(l_{m}\right)}\left(z^{2}\right) Q^{\prime}(z)$ have to have the same orders so that the centers of symmetry align. Thus, from (39), $\operatorname{Order}\left[B_{0,0}^{\left(l_{m}\right)}\left(z^{2}\right)\right]=2$ and $\operatorname{Order}\left[B_{0,1}^{\left(i_{m}\right)}\left(z^{2}\right)\right]=0$. Similarly, by considering the second equation of (38), $\operatorname{Order}\left[B_{1,0}^{\left(l_{m}\right)}\left(z^{2}\right)\right]=(4 l+4)$ and $\operatorname{Order}\left[B_{1.1}^{\left(l_{m}\right)}\left(z^{2}\right)\right]=$ $(4 l+2)$. In summary, to obtain a symmetric pair $\left[P^{\prime}(z)\right.$, $\left.Q^{\prime}(z)\right]$ with orders $(m-4 l-4)$ and $(m-4 l-2)$ from a symmetric pair $\left[P_{r}(z), Q_{m}(z)\right]$ with orders $(m-4 l-$ 2 ) and $m$, all the elements of the building block $\boldsymbol{B}^{\left(l_{m}\right)}\left(z^{2}\right)$ are symmetric polynomials and moreover, the orders of the elements can be summarized as

$$
\operatorname{Order}\left[\boldsymbol{B}^{\left(l_{m}\right)}\left(z^{2}\right)\right]=\left[\begin{array}{ll}
2 & 0 \\
4 l+4 & 4 l+2
\end{array}\right]
$$

We now propose a particular form for $\boldsymbol{B}^{\left(l_{m}\right)}\left(z^{2}\right)$ and through the "downward recursion"' on the pair $\left[P_{r}(z)\right.$, $\left.Q_{m}(z)\right]$ show that this particular form of $\boldsymbol{B}^{\left(l_{m}\right)}\left(z^{2}\right)$ is indeed sufficient to cover all Type $B$ pairs. The proposed form is

$$
\boldsymbol{B}^{(/ m)}(z)=\left[\begin{array}{ll}
1+z^{-1} & 1 \\
T(z) & U(z)
\end{array}\right]
$$

where $T(z)$ and $U(z)$ are symmetric polynomials of orders $(2 l+2)$ and $(2 l+1)$, respectively, i.e., $T(z)=$ $\Sigma_{j=0}^{2 l+2} t_{j} z^{-j}$ and $U(z)=\Sigma_{j=0}^{2 l+1} u_{j} z^{-j}$. We assume that $t_{0}$ $=u_{0}=1$ (see justification later). From (41), the condition "det $\boldsymbol{B}^{\left(l_{m}\right)}(z)=$ delay" is equivalent to

$$
\left(1+z^{-1}\right) U(z)-T(z)=c z^{-(1+1)} \text {. }
$$

Equating the like powers of $z$ in (42), we obtain

$$
\left\{\begin{array}{l}
t_{0}=u_{0}, \\
t_{k}=u_{k-1}+u_{k}, \quad 1 \leq k \leq l, \\
c=2 u_{l}-t_{l+1} \neq 0 .
\end{array}\right.
$$

Thus, any choices of $u_{k}$ and $t_{k}$ that satisfy (43) guarantee that $\operatorname{det} \boldsymbol{B}^{\left(l_{n}\right)}(z)=c z^{-(l+1)}$. In the synthesis procedure, we show how to find $u_{k}, 1 \leq k \leq l$ such that the orders of $\left[P^{\prime}(z), Q^{\prime}(z)\right]$ are $(m-4 l-4)$ and $(m-4 l$ $-2)$. Then det $\boldsymbol{B}^{\left(l_{m}\right)}(z)$ is forced to be a delay by choosing $t_{k}$ as in (43), and the synthesis procedure is completed. 
Noticing from (43) that $t_{l+1}$ is arbitrary, we assume that it is chosen such that $c \neq 0$. From Fig. 11(a), we then have

$$
\left[\begin{array}{l}
P^{\prime}(z) \\
Q^{\prime}(z)
\end{array}\right]=\frac{z^{2(l+1)}}{c}\left[\begin{array}{cc}
U\left(z^{2}\right) & -1 \\
-T\left(z^{2}\right) & 1+z^{-2}
\end{array}\right]\left[\begin{array}{l}
P_{r}(z) \\
Q_{m}(z)
\end{array}\right]
$$

Since we are interested in obtaining causal filters $P^{\prime}(z)$, $Q^{\prime}(z)$, we should choose $U(z)$ such that

$$
U\left(z^{2}\right) P_{r}(z)-Q_{m}(z)=z^{-2(l+1)} \alpha_{0}(z)
$$

and

$$
-T\left(z^{2}\right) P_{r}(z)+\left(1+z^{-2}\right) Q_{m}(z)=z^{-2(l+1)} \alpha_{1}(z)
$$

for some causal FIR $\alpha_{0}(z), \alpha_{1}(z)$. Now, forcing the condition (45) automatically guarantees (46) because (45) implies

$$
\begin{aligned}
\left(1+z^{-2}\right) U\left(z^{2}\right) P_{r}(z)-\left(1+z^{-2}\right) Q_{m}(z) \\
=z^{-2(l+1)}\left(1+z^{-2}\right) \alpha_{0}(z)
\end{aligned}
$$

which, in view of (42), simplifies to the form (46). It therefore remains only to satisfy (45). For convenience, write $P_{r}(z)$ and $Q_{m}(z)$ in the polyphase forms $P_{r}(z)=$ $P_{r, 0}\left(z^{2}\right)+z^{-1} P_{r, 1}\left(z^{2}\right)$ and $Q_{m}(z)=Q_{m, 0}\left(z^{2}\right)+$ $z^{-1} Q_{m, 1}\left(z^{2}\right)$, respectively. Then (45) breaks into two equations:

$$
\begin{aligned}
& U(z) P_{r, 0}(z)-Q_{m, 0}(z)=z^{-(l+1)} \beta_{0}(z) \\
& U(z) P_{r, 1}(z)-Q_{m, 1}(z)=z^{-(l+1)} \beta_{1}(z)
\end{aligned}
$$

for some causal FIR $\beta_{0}(z), \beta_{1}(z)$. We now show that (47) implies (48) automatically because of the constraint (36). For this, note that $m-4 l-2$ is the degree of $P_{r}(z)$ so that $m / 2-2 l-1>0$, which implies $m / 2-l-1$ $\geq l+1$. As a result, (36) implies, in particular,

$$
P_{r .0}(z) Q_{m, 1}(z)-P_{r .1}(z) Q_{m, 0}(z)=z^{-(l+1)} \delta_{0}(z)
$$

for some causal FIR $\delta_{0}(z)$. Multiplying both sides of (47) by $P_{r, 1}(z)$ and substituting (49) results in

$$
U(z) P_{r, 0}(z) P_{r, 1}(z)-P_{r, 0}(z) Q_{m, 1}(z)=z^{-(l+1)} \delta_{1}(z)
$$

for some causal FIR $\delta_{1}(z)$. Since $p_{r, 0} \neq 0$ by assumption, (50) implies (48) proving that (47) implies (48).

Summarizing, we can ensure that we obtain a causal reduced-degree Type B pair $\left[P^{\prime}(z), Q^{\prime}(z)\right]$ in Fig. 11(a) simply by satisfying (47)! The condition (47) can be satisfied by choosing the coefficients $u_{n}, 1 \leq n \leq l$ of $U(z)$ such that $U(z) P_{r, 0}(z)=Q_{m, 0}(z)+z^{-(l+1)} \beta_{0}(z)$. This can be written as a triangular set of equations

$$
\left[\begin{array}{cccc}
p_{r, 0} & 0 & \cdots & 0 \\
p_{r, 2} & p_{r, 0} & \cdots & 0 \\
\vdots & \vdots & \ddots & \vdots \\
p_{r, 2 l} & p_{r, 2 l-2} & \cdots & p_{r, 0}
\end{array}\right]\left[\begin{array}{c}
1 \\
u_{1} \\
\vdots \\
u_{l}
\end{array}\right]=\left[\begin{array}{c}
q_{m, 0} \\
q_{m, 2} \\
\vdots \\
q_{m, 2 l}
\end{array}\right] .
$$

The first equation above is automatically satisfied because of (33). The remaining equations can be satisfied by solving for a unique set $u_{1}, \cdots, u_{l}$ because $p_{r, 0} \neq 0$ in (51).

In summary, we first find $U(z)$ satisfying $(51)$ and then find $T(z)$ using (43). Consequently, $\boldsymbol{B}^{\left(l_{m}\right)}(z)$ is determined. To be able to apply the same synthesis procedure on the Type B pair $\left[P^{\prime}(z), Q^{\prime}(z)\right]$, its coefficients have to satisfy a condition analogous to (33). Denoting the coefficients of $P^{\prime}(z)$ and $Q^{\prime}(z)$ by $p_{j}^{\prime}$ and $q_{j}^{\prime}$, this condition is satisfied by a scale factor $\alpha_{m}$ if $p_{0}^{\prime} \neq 0$ and $q_{0}^{\prime} \neq 0$. The complete building block for the case where $p_{0}^{\prime} \neq 0$ and $q_{0}^{\prime} \neq 0$ is shown in Fig. 11(b). Clearly, (33) cannot be satisfied by just a scale factor if $p_{0}^{\prime}=0$ or $q_{0}^{\prime}=0$. We now elaborate on the remedy for this case ( $p_{0}^{\prime}=0$ or $q_{0}^{\prime}$ $=0$ ).

The remedy is to choose the extra freedom $t_{l+1}$ such that $q_{0}^{\prime} \neq 0$. With $q_{0}^{\prime} \neq 0$, we show now that if $p_{0}^{\prime}=0$, then we can always pull out a delay of the specific form $z^{-2 K_{m}}$ [as demonstrated in Fig. 11(c)] such that the first coefficient of $V_{0}(z)$ is nonzero. (Having done so, the pair $\left[V_{1}(z), Q^{\prime}(z)\right]$ is a causal Type B pair. We can therefore repeat the above order-reduction process.) For this, represent the pair $P^{\prime}(z)$ and $Q^{\prime}(z)$ in polyphase form:

$$
\left[\begin{array}{l}
P^{\prime}(z) \\
Q^{\prime}(z)
\end{array}\right]=\left[\begin{array}{ll}
P_{0}^{\prime}\left(z^{2}\right) & P_{1}^{\prime}\left(z^{2}\right) \\
Q_{0}^{\prime}\left(z^{2}\right) & Q_{1}^{\prime}\left(z^{2}\right)
\end{array}\right]\left[\begin{array}{c}
1 \\
z^{-1}
\end{array}\right]=\boldsymbol{E}^{\prime}\left(z^{2}\right)\left[\begin{array}{c}
1 \\
z^{-1}
\end{array}\right] \text {. }
$$

We know that the determinant of $\boldsymbol{E}^{\prime}(z)$ is a delay by our above construction of the pair $\left[P^{\prime}(z), Q^{\prime}(z)\right]$. Thus,

$$
P_{0}^{\prime}(z) Q_{1}^{\prime}(z)-P_{1}^{\prime}(z) Q_{0}^{\prime}(z)=z^{-(m / 2-2 l-2)} .
$$

If, for some reason, we have $p_{0}^{\prime}=0$, then $P_{0}^{\prime}(z) Q_{1}^{\prime}(z)$ has the form $z^{-1} \alpha(z)$ where $\alpha(z)$ is some causal FIR system. Because of $(52 \mathrm{~b})$, this implies that $P_{1}^{\prime}(z) Q_{0}^{\prime}(z)$ has this form as well. Since $q_{0}^{\prime} \neq 0$, this necessarily implies $p_{1}^{\prime}=0$. In other words, $p_{0}^{\prime}=0$ implies $p_{1}^{\prime}=0$ as well. More generally, it is easily verified based on this type of argument that if $p_{n}^{\prime}=0$ for $0 \leq n \leq K$ with $p_{K+1}^{\prime} \neq 0$, then $K$ is odd. This means we can factorize $P^{\prime}(z)$ as $P^{\prime}(z)$ $=z^{-2 K_{m}} V_{0}(z)$.

With this, Fig. 11(a) becomes Fig. 11(c) where the pair $\left[V_{0}(z), Q^{\prime}(z)\right]$ now satisfies $v_{0,0} \neq 0, q_{0}^{\prime} \neq 0$. We now insert a scale factor $\alpha_{m}$ as shown in Fig. 11(c) such that the pair $\left[V_{1}(z), Q^{\prime}(z)\right]$ in Fig. $11(\mathrm{c})$ is a Type B pair with orders $\left(m-4 l-4-4 K_{m}\right)$ and $(m-4 l-2)$, respectively. Moreover, it satisfies $v_{1,0}=q_{0}^{\prime} \neq 0$, which is analogous to (33). Consequently, the order reduction 


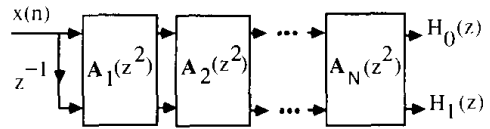

Fig. 12. The lattice structure for Type B system.

process can now be repeated to obtain a Type B structure as shown in Fig. 12 where $\boldsymbol{A}_{j}\left(z^{2}\right)$ is shown in Fig. 11(c). Note that Fig. 11(b) is the same as Fig. 11(c) when $K_{m}=$ 0 .

The only remaining question to be answered is whether or not we can choose $t_{l+1}$ such that $q_{0}^{\prime} \neq 0$ (and $c \neq 0$ ). The answer is in the affirmative. From (44),

$$
q_{0}^{\prime}=q_{m, 2 l+2}+q_{m, 2 l+4}-\sum_{j=0}^{l} t_{j} p_{r, 2 l+2-2 j}-t_{l+1} p_{r, 0} \text {. }
$$

It is clear from the above equation and the assumption $p_{r, 0} \neq 0$, that we have to choose $t_{i+1}$ such that

$$
t_{l+1} \neq \frac{1}{p_{r, 0}}\left[q_{m .2 l+2}+q_{m .2 l+4}-\sum_{j=0}^{l} t_{j} p_{r, 2 l+2-2 j}\right] \text {. }
$$

We summarize the synthesis procedure as follows.

Given a Type B pair $\left[P_{r}(z), Q_{m}(z)\right]$ of order $(m-4 l$ -2 ) and $m$ (where $m$ is even) which satisfies (33), do the following.

- Compute $u_{n}, 0 \leq n \leq l$ using (51) and $t_{n}$ using (43). Choose the coefficient $t_{l+1}$ such that $q_{0}^{\prime} \neq 0$. This determines $\boldsymbol{B}^{\left(l_{m}\right)}(z)$.

- If $p_{0}^{\prime} \neq 0$, then scale the pair $\left[P^{\prime}(z), Q^{\prime}(z)\right]$ to satisfy $p_{0}^{\prime}=q_{0}^{\prime}$. The complete building block is shown in Fig. $11(\mathrm{~b})$.

- If $p_{0}^{\prime}=0$, then pull out the delay $z^{-2 K_{m}}$, with appropriate $K_{m}$. Then scale the pair $\left[V_{0}(z), Q^{\prime}(z)\right]$ to obtain $\left[V_{1}(z), Q^{\prime}(z)\right]$ which satisfies $v_{1.0}=q_{0}^{\prime}$. The resulting block is shown in Fig. 11(c).

Since Fig. $11(\mathrm{~b})$ is a special case $\left(K_{m}=0\right)$ of Fig. 11(c), therefore, the most general building block $\boldsymbol{A}_{j}(z)$ of the overall Type B structure (in Fig. 12) is as in Fig. $11(\mathrm{c})$.

Example 4.1: The building blocks in Fig. 12 have the two added freedoms, namely, $l_{m}$ and $K_{m}$. To simplify the optimization process, we choose $l_{m}=K_{m}=0$ for all blocks $\boldsymbol{A}_{j}(z)$ in Fig. 12. The resulting structure to be used in the optimization procedure is shown in Fig. 13 where

$$
B_{m}^{(0)}(z)=\left[\begin{array}{ll}
1+z^{-1} & 1 \\
1+a_{m} z^{-1}+z^{-2} & 1+z^{-1}
\end{array}\right] .
$$

Even though the structure has $2 N$ parameters $\alpha_{m}, a_{m}, 1 \leq$ $m \leq N$, there are only $N$ degrees of freedom. This is evident from the deviation of the structure above. Thus, the parameters $a_{m}$ are precisely the free parameters $t_{l+1}$ used in (43), and can be chosen arbitrarily. In the design example, we fixed the parameters $a_{m}$ to be equal to 64 and optimized $\alpha_{m}$. The choice $a_{m}=64$ is, however, entirely

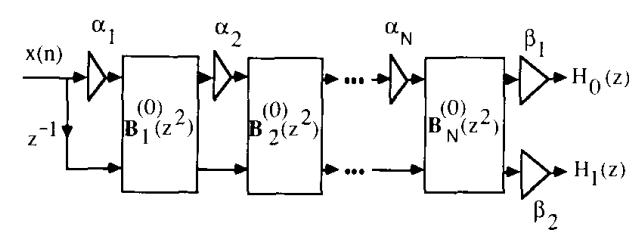

Fig. 13. The lattice structure for Type B system used in the optimization procedure. Here $l_{n}=0$ and $K_{n}=0$.

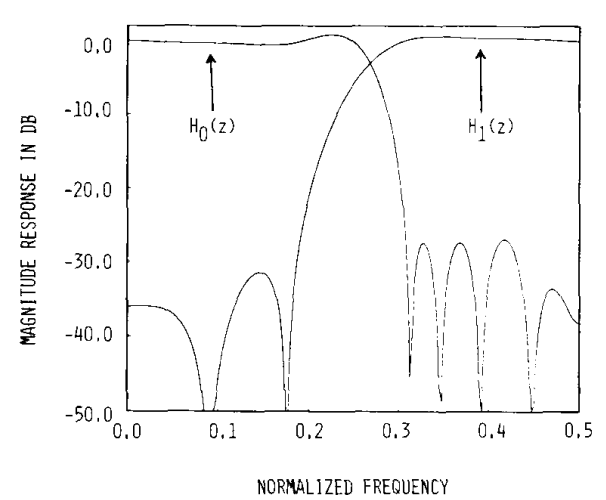

(a)

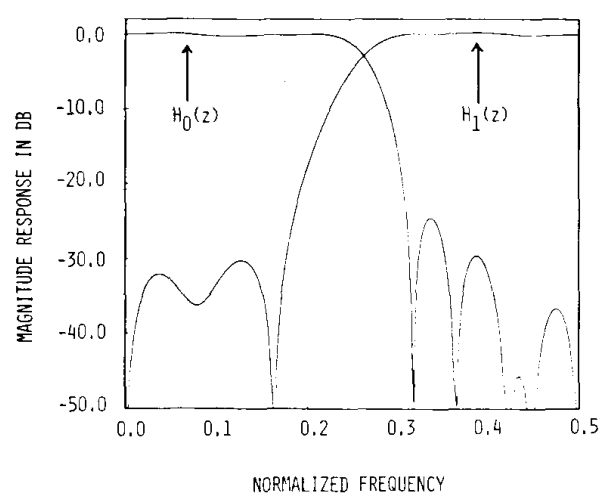

(b)

Fig. 14. (a) Example 4.1: Magnitude response plots for the optimized analysis filters (Type B). The filter lengths are 23 and 25 . respectively. (b) Example 4.1: Magnitude response plots for the optimized analysis filters (Type A). The filter lengths are 22 each.

arbitrary and not based on any engineering judgement. At this point in time, we do not have any indication that a particular set of $a_{m}$ should be preferred to another.

We have $N=11$, and the transition bandwidth $\Delta f=$ 0.1 in the example. The orders of $H_{0}(z)$ and $H_{1}(z)$ are thus 22 and 24 , respectively. We minimize the objective function described in (30) using [15]. The magnitude responses of the optimized analysis filters are shown in Fig. 14(a). The lattice coefficients $\alpha_{m}$ and the impulse responses of both filters are summarized in Table $\mathrm{V}$. The complexity of this structure can be readily computed by noting that there is only one multiplier per building block in Fig. 13, namely, $\alpha_{m}\left(a_{m}=64\right.$ can be realized by shifting). Moreover, each building block can be implemented with 5 addition operations. Thus, with a factor of 2 saving 
TABLE V

LatTice CoefFicients and IMPULSE Responses of The OPTIMIZED ANALYSIS FILTERS IN EXAMPLE 4.1. (SSEE) $\beta_{1}=7.81221 \times 10^{-15}, \beta_{2}=$ $1.37742 \times 10^{-18}$

\begin{tabular}{|c||r|r|r|}
\hline \hline$m$ & Lattice Coeff. $\alpha_{2 m+1}$ & \multicolumn{1}{|c|}{ Filter Coefficients $h_{0, m}$} & \multicolumn{1}{|c|}{ Filter Coefficients $h_{1, m}$} \\
\hline 0 & -0.37779962858106 & $5.9250950404227 \times 10^{-3}$ & $1.0446957436687 \times 10^{-8}$ \\
\hline 1 & 28.980740681587 & $-1.5683167986894 \times 10^{-2}$ & $-2.765211453693 \times 10^{-6}$ \\
\hline 2 & -47.817981025871 & $4.5594028972112 \times 10^{-3}$ & $8.2562648947712 \times 10^{-6}$ \\
\hline 3 & -26.815036810932 & $3.6047559927372 \times 10^{-2}$ & $-1.3369902864879 \times 10^{-6}$ \\
\hline 4 & -1012355.8728888 & $-2.2314449066179 \times 10^{-2}$ & $-3.7402147190058 \times 10^{-3}$ \\
\hline 5 & -1.0003784977510 & $-4.2444851133377 \times 10^{-2}$ & $9.9426163128459 \times 10^{-4}$ \\
\hline 6 & 0.00041704202379460 & $4.0981175348012 \times 10^{-2}$ & $5.2822531950793 \times 10^{-4}$ \\
\hline 7 & -164.81675604153 & $6.3735691673040 \times 10^{-3}$ & $-3.1883222956165 \times 10^{-2}$ \\
\hline 8 & -7501.9622674952 & $-8.7080719666949 \times 10^{-2}$ & $-1.1276359972347 \times 10^{-3}$ \\
\hline 9 & -1.1059836252728 & $-6.4640370944037 \times 10^{-2}$ & $9.5100122977005 \times 10^{-2}$ \\
\hline 10 & 0.10978902673135 & $3.1314592005381 \times 10^{-1}$ & $-9.5195769252465 \times 10^{-4}$ \\
\hline 11 & & $5.6912350680866 \times 10^{-1}$ & $-3.1042174432319 \times 10^{-1}$ \\
\hline 12 & & & $5.0039707172893 \times 10^{-1}$ \\
\hline
\end{tabular}

TABLE VI

Comparison Between TyPe A and Type B PR Systems for the Design Example. Here $\delta_{1}$ and $\delta_{2}$ Denote the Peak-Ripple Sizes in Passband and Stopband, Respectively. BOTh TyPES ReQuire 6.5 MPU'S For the Analysis Bank. The Type A System Requires 17.5 APU's Whereas THE TyPE B SYSTEM REQUIRES 27.5 APU'S

\begin{tabular}{|c||c|c|c|c|}
\hline \multicolumn{1}{|c||}{} & \multicolumn{2}{c|}{ Type A } & \multicolumn{2}{c|}{ Type B } \\
\hline & $H_{0}(z)$ & $H_{1}(z)$ & $H_{0}(z)$ & $H_{1}(z)$ \\
\hline Filter length & 22 & 22 & 23 & 25 \\
\hline$\delta_{1}$ & $2.46 \times 10^{-2}$ & $2.60 \times 10^{-2}$ & $3.27 \times 10^{-2}$ & $3.49 \times 10^{-2}$ \\
\hline$\delta_{2}$ & $5.92 \times 10^{-2}$ & $3.07 \times 10^{-2}$ & $4.49 \times 10^{-2}$ & $2.67 \times 10^{-2}$ \\
\hline
\end{tabular}

in the decimation ratio, the complexity of the analysis bank is $(N+2) / 2=(11+2) / 2=6.5 \mathrm{MPU}$ and $5 N / 2$ $=27.5$ APU.

The fact that we can obtain a linear-phase PR pair with even orders for the filters and with both filters symmetric is not entirely surprising. Indeed, procedures for design of QMF banks with linear-phase even-order filters have been presented in [16], which are approximately PR. The results we have presented above, however, have exact PR property. It should be noticed that Johnston's filters [1], [22] were designed for the even-length case, and therefore are not suitable for comparison to the Type B PR system reported in this paper.

\section{A. Comparison of Type A and Type B Systems}

To compare the two types of PR systems reported in this paper, we design a Type A pair with the same number of sections and transition bands as the Type B pair in Example 4.1 , i.e., $N=11$ and $\Delta f=0.1$. The frequency response of this Type A pair is shown in Fig. 14(b). The lengths of the Type $A$ analysis filters are 22 . The passband and stopband errors of $H_{0}(z)$ and $H_{1}(z)$ for both PR systems are summarized in Table VI. The number of MPU's and APU's required for both types are also included in the table. From the table, it is evident that both systems have nearly identical filtering performance, and require the same number of MPU's. Type B systems, however, require a larger number of APU's.

\section{Concluding Remarks}

We have described two perfect-reconstruction structures for the two-channel QMF bank, free of aliasing and distortions of any kind, in which the analysis filters have linear phase. The first lattice structure relates closely to the LPC lattice, and it covers most of the SAOO LP PR FIR pairs of filters. The second lattice structure covers all SSEE LP PR FIR pairs of filters. Furthermore, the perfect-reconstruction and linear-phase properties of these filters are structurally enforced (in spite of quantization of the lattice coefficients). Design examples are given to verify the theory.

\section{APPENDIX A Singularity Issues}

Given the MIP $\left[T_{N}(z), U_{N}(z)\right]$, consider the synthesis procedure of the lattice of Fig. 5 again. The synthesis procedure is to recursively compute lower order MIP's according to the relation

$$
\left[\begin{array}{l}
T_{m-1}^{\prime}(z) \\
z^{-1} U_{m-1}^{\prime}(z)
\end{array}\right]=\left[\begin{array}{cc}
1 & -k_{m} \\
-k_{m} & 1
\end{array}\right]\left[\begin{array}{c}
T_{m}^{\prime}(z) \\
U_{m}^{\prime}(z)
\end{array}\right]
$$

with $T_{N}^{\prime}(z)=T_{N}(z)$ and $U_{N}^{\prime}(z)=U_{N}(z)$. The coefficient $k_{m}$ is computed as

$$
k_{m}=\frac{t_{m . m}^{\prime}}{t_{m .0}^{\prime}}
$$

The unprimed polynomials in (24) are essentially scaled versions of the primed ones in (A1). The inverse of the relation (A1) is

$$
\left[\begin{array}{c}
T_{m}^{\prime}(z) \\
U_{m}^{\prime}(z)
\end{array}\right]=\frac{1}{1-k_{m}^{2}}\left[\begin{array}{cc}
1 & k_{m} \\
k_{m} & 1
\end{array}\right]\left[\begin{array}{l}
T_{m-1}^{\prime}(z) \\
z^{-1} U_{m-1}^{\prime}(z)
\end{array}\right]
$$

which results in the lattice structure of Fig. 5 (except for the scale factor $1-k_{m}^{2}$ ), upon repeated application of the above recursion.

Assume now that, at some stage, $k_{m}^{2}=1$. This means that the recursion (A1) would give $T_{m-1}^{\prime}(z)=$ $\pm z^{-1} U_{m-1}^{\prime}(z)$. However, the inverse relation (A3) is now meaningless [and so is the scaled inverse relation (23)] because the $2 \times 2$ matrix in (A1) is singular. This means that we cannot get back $\left[T_{m}(z), U_{m}(z)\right]$ by starting from $\left[T_{m-1}(z), U_{m-1}(z)\right]$, i.e., there simply does not exist a lattice of the form of Fig. 5 in this case. (Notice that, under this situation, an attempt to use (23) would lead to the conclusion $T_{m}(z)= \pm U_{m}(z)$, which of course is not necessary for " $k_{m}^{2}=1$ " to happen.)

Next, conversely, suppose we have the lattice of Fig. 5 already synthesized for some $\operatorname{MIP}\left[T_{N}(z), U_{N}(z)\right]$, and we replace $k_{m}$ with unity for some $m$. This means from (23) (which now holds!) that $T_{m}(z)=U_{m}(z)$, which in turn means that $T_{N}(z)$ and $U_{N}(z)$ share the common factor $T_{m}(z)$. An attempt to synthesize this $\left[T_{N}(z), U_{N}(z)\right]$ using (A1) will once again bring about the situation $k_{m}^{2}=$ 1 , but the synthesis procedure cannot be carried out beyond this point. 


\section{Appendix B}

EXISTENCE OF $z_{m}$

As explained in Section III, a singularity situation can be avoided by using the modified recursion (25a), (25b), with $k_{m}$ as in (26). We can find a $z_{m}$ such that $k_{m}^{2} \neq 1$ (and $\left.k_{m}^{2}<\infty\right)$ as long as none of the three polynomials

$$
\begin{aligned}
& P_{1}(z)=T_{m}(z)-U_{m}(z) \\
& P_{2}(z)=T_{m}(z)+U_{m}(z) \\
& P_{3}(z)=U_{m}(z)
\end{aligned}
$$

is identically zero. If $\left[H_{0}(z), H_{1}(z)\right]$ is such that one of these polynomials is identically zero for some $m$, what does this signify?

Assume that we start with $m=N$ and carry out (25) until we arrive at the situation when one of the three polynomials $P_{1}(z), P_{2}(z)$, and $P_{3}(z)$ is zero. If $P_{1}(z) \equiv 0$ or $P_{2}(z) \equiv 0$, this means $T_{m}(z)= \pm U_{m}(z)$, which in turn means (see Fig. 15) that $T_{m}(z)$ is a common factor between $H_{0}(z)$ and $H_{1}(z)$. If, on the other hand, $U_{m}(z)$ $=0$, this means $T_{m}(z)=0$ as well since $T_{m}(z)=\hat{U}_{m}(z)$, and this implies $H_{0}(z)=H_{1}(z) \equiv 0$.

In conclusion, if $H_{0}(z)$ and $H_{1}(z)$ are not identically zero, and do not share a common factor, then there will exist a $z_{m}$ such that $k_{m}^{2} \neq 1$ for every $m$.

\section{Appendix C \\ Existence of COMmon Factors}

Consider the QMF bank of Fig. 1 where the four filters are FIR. If $H_{0}(z)$ and $H_{1}(z)$ have a common zero on the unit circle of the form $\left(1-z^{-1} e^{j \omega_{0}}\right)$, then an input $x(n)$ $=e^{j \omega_{0} n}$ cannot be "perfectly reconstructed." In fact, if $H_{0}(z)$ and $H_{1}(z)$ share any common factor $\left(1-z^{-1} \alpha\right)$, then an exponential input $x(n)=\alpha^{n}$ would produce an identically zero output $\hat{x}(n)$, violating the PR property. Without loss of generality, we can therefore assume that $H_{0}(z)$ and $H_{1}(z)$ do not have any common factors.

\section{APPENDIX D}

Let the FIR filters $H_{0}(z)$ and $H_{1}(z)$ be related as $H_{1}(z)$ $=H_{0}(-z)$. Then the polyphase-component matrix $\boldsymbol{E}(z)$ has the form

$$
\boldsymbol{E}(z)=\left[\begin{array}{cc}
E_{00}(z) & E_{01}(z) \\
E_{00}(z) & -E_{01}(z)
\end{array}\right]
$$

so that det $\boldsymbol{E}(z)=-2 E_{00}(z) E_{01}(z)$. If this has to be a delay, then we must have $E_{00}(z)=c_{0} z^{-n 0}$ and $E_{01}(z)=$ $c_{1} z^{-n !}$ for some integers $n_{0}, n_{1} \geq 0$. This means $H_{0}(z)=$ $c_{0} z^{-2 n)}+c_{1} z^{-(2 n+1)}$ which is a very restricted class of transfer functions indeed. In order to obtain a good PR pair, it is therefore essential to remove the restriction $H_{1}(z)=H_{0}(-z)$.

\section{Appendix $\mathrm{E}$}

$M$-Channel Generalizations (Even $M$, SAOO)

Let $H_{k}(z)$ be the analysis filters of odd order $N_{k}-1$, $0 \leq k \leq M-1$. Furthermore, we assume that the first

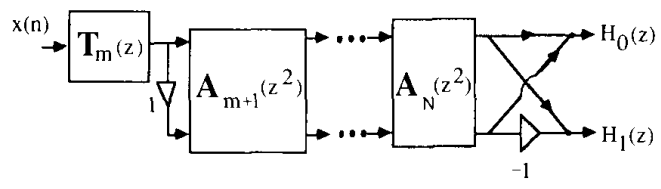

Fig. 15. Pertaining to Appendix B.

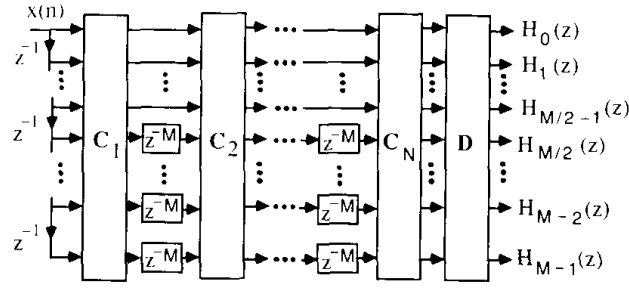

Fig. 16. The lattice structure for the $M$-channel SAOO LP PR QMF bank (even $M$ ).

$M / 2$ filters are symmetric and the last $M / 2$ ones are antisymmetric. Consider $H_{k}(z)$ and $H_{M-1-k}(z)$ for $0 \leq k$ $\leq M / 2-1$. Since they have odd orders and opposite symmetries, they constitute an SAOO pair which we discussed in Section III. Thus, one obvious extension from the two-channel SAOO case to the $M$-channel case (even $M, \mathrm{SAOO}$ ) is that we consider $M / 2 \mathrm{SAOO}$ pairs in such a way that they do not interact with each other. In other words, each SAOO pair $\left[H_{k}(z), H_{M-1-k}(z)\right], 0 \leq k \leq$ $M / 2-1$ is realized as in Fig. 9 with $z^{-2}$ replaced by $z^{-M}$. These pairs can then be appropriately combined. The overall PR structure is shown in Fig. 16 where the building blocks are

$$
\begin{gathered}
C_{i}=\left[\begin{array}{lll}
I & & \Theta \\
P_{0} & \Theta P_{0} & I
\end{array}\right], \\
D=\left[\begin{array}{cccccccc}
1 & 0 & \cdots & 0 & 0 & \cdots & 0 & 1 \\
0 & 1 & \cdots & 0 & 0 & \cdots & 1 & 0 \\
\vdots & \vdots & \ddots & \vdots & \vdots & . & \vdots & \vdots \\
0 & 0 & \cdots & 1 & 1 & \cdots & 0 & 0 \\
0 & 0 & \cdots & 1 & -1 & \cdots & 0 & 0 \\
\vdots & \vdots & \ddots & \vdots & \vdots & \ddots & \vdots & \vdots \\
0 & 1 & \cdots & 0 & 0 & \cdots & -1 & 0 \\
1 & 0 & \cdots & 0 & 0 & \cdots & 0 & -1
\end{array}\right]
\end{gathered}
$$

where

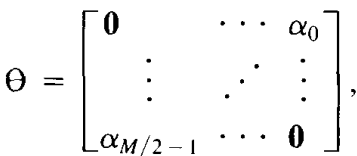

$$
\begin{aligned}
& \boldsymbol{P}_{0}=\left[\begin{array}{ccc}
\mathbf{0} & \cdots & 1 \\
\vdots & \ddots & \vdots \\
1 & \cdots & 0
\end{array}\right] .
\end{aligned}
$$


This structure (Fig. 16) yields PR odd-order linear-phase filters with opposite symmetry. We, however, do not have the most general structure for this purpose, at this time. This is currently under study.

\section{REFERENCES}

[1] R. E. Crochiere and L. R. Rabiner, Multirate Digital Signal Processing. Englewood Cliffs, NJ: Prentice-Hall, 1983.

12] M. J. T. Smith and T. P. Barnwell, III, "Exact reconstruction techniques for tree structured subband coders," IEEE Trans. Acoust. Speech, Signal Processing, vol. ASSP-34, pp. 434-441, June 1986.

[3] F Mintzer, "Filters for distortion free two-band multirate filte banks," IEEE Trans. Acoust., Speech, Signal Processing, vol. ASSP33, pp. 626-630, June 1985.

14] P. P. Vaidyanathan and P. Q. Hoang, "Lattice structures for optimal design and robust implementation of two-channel perfect-reconstruction QMF banks,' IEEE Trans. Acoust., Speech, Signal Processing, vol. 36, pp. 81-94, Jan. 1988.

15] P. P. Vaidyanathan, "Theory and design of $M$ channel maximally decimated quadrature mirror filters with arbitrary $M$, having perfect reconstruction property," IEEE Trans. Acoust., Speech, Signal Processing, vol. ASSP-35, pp. 476-492, Apr. 1987.

16] M. Vetterli, "A theory of multirate filter banks," IEEE Trans. Acoust., Speech, Signal Processing, vol. ASSP-35, pp. 356-372, Mar. 1987.

[7] P. P. Vaidyanathan, Z. Doganata, and T. Q. Nguyen, "More results on the perfect reconstruction problem in $M$-band, parallel QMF banks," in Proc. ISCAS, May 1987.

18] J. D. Markel and A. H. Gray, Jr., Linear Prediction of Speech. New York: Springer-Verlag, 1976

[9] L. R. Rabiner and B. Gold. Theory and Application of Digital Signal Processing. Englewood Cliffs, NJ: Prentice-Hall, 1975

110] M. Vetterli, "Filter banks allowing perfect reconstruction," Signal Processing, vol. 10, no. 3, pp. 219-244, Apr. 1986.

[11] M. G. Bellanger, G. Bonnerot, and M. Coudreuse, "Digital filtering by polyphase network: Application to sample-rate alternation and filter banks," IEEE Trans. Acoust., Speech, Signal Processing, vol. ASSP-24, pp. 109-114. Apr. 1976

[12] J. Makhoul. "Stable and efficient lattice methods for linear predic tion." IEEE Trans. Acoust., Speech, Signal Processing, vol. ASSP25, pp. 423-428, Oct. 1977.

[13] P. P. Vaidyanathan and S. K. Mitra, "A unified structural interpretation of some well-known stability-test procedures for linear systems," Proc. IEEE, vol. 75, pp. 478-497, Apr. 1987.

[14] _ , "Discrete version of Richards' theorem and application to cascaded lattice realization of digital filter transfer matrices and functions," IEEE Trans. Circuits Syst., vol. CAS-33, pp. 26-34, Jan. 1986

[15] IMSL: A Fortran Mathematical Library

[16] C. R. Galand and H. J. Nussbaumer, "New quadrature mirror filter structures," IEEE Trans. Acoust., Speech. Signal Processing. vol. ASSP-32, pp. 522-531, June 1984.

[17] J. Makhoul, "A class of all-zero lattice digital filters: Properties and applications," IEEE Trans. Acoust., Speech, Signal Processing, vol ASSP-26. pp. 304-314, Aug. 1978.

$118]$ Z. Doganata and P. P. Vaidyanathan, "On one-multiplier implemen tations of FIR lattice structures," IEEE Trans. Circuits Syst., vol CAS-34, pp. 1608-1609, Dec. 1987.

[19] P. P. Vaidyanathan. "Quadrature mirror filter banks, $M$-band extensions and perfect-reconstruction techniques," IEEE ASSP Mag., vol 4, pp. 4-20. July 1987
[20] T. Q. Nguyen and P. P. Vaidyanathan, "Perfect reconstruction QMF structures which yield linear phase FIR analysis filters," presented a the IEEE Int. Conf. Circuits Syst., Finland, May 1988.

[21] P. P. Vaidyanathan, "On power-complementary FIR filters," IEEE Trans. Circuits Syst, vol. CAS-32, pp. 1308-1310, Dec. 1985.

[22] J. D. Johnston, "A filter family designed for use in quadrature mirror filter banks," in Proc. Int. Conf. Acoust., Speech. Signal Processing. Apr. 1980, pp. 291-294.

[23] V. K. Jain and R. E. Crochiere, "Quadrature mirror filter design in the time domain," IEEE Trans. Acoust., Speech, Signal Processing. pp. 353-361, Apr. 1984

[24] D. Esteban and C. Galand, "Application of quadrature mirror filters to split-band voice coding schemes," in Proc. IEEE Int. Conf. ASSP. Hartford. CT, May 1977, pp. 191-195.

[25] M. Vetterli and D. LeGall, "Analysis and design of perfect reconstruction filter banks satisfying symmetry constraints, "in Proc. 22 nd Conf. Inform. Sci. Syst., Princeton, NJ, Mar. 1988, pp. 670-675.

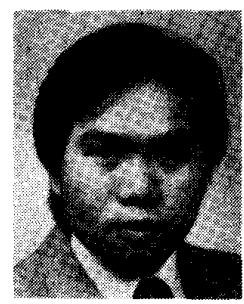

Truong Q. Nguyen ( $\mathrm{S}^{\circ} 86$ ) was born in Saigon. Vietnam, on November 2,1962 . He received the B.S. (hons.) and M.S. degrees from California Institute of Technology in electrical engineering in 1985 and 1986, respectively, and is currently pursuing the $\mathrm{Ph}$.D. degree at the same institution.

During the academic year 1986-1987 he was a recipient of a fellowship from Aerojet Dynamics. for advanced studies. His main research interests are in digital signal processing, multirate filter bank systems, and filter design.

Mr. Nguyen is a member of Tau Beta Pi and Eta Kappa Nu.

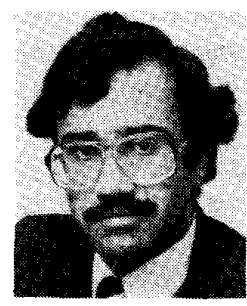

P. P. Vaidyanathan ( $\left.\mathrm{S}^{\prime} 80-\mathrm{M}^{\prime} 83-\mathrm{SM}^{\prime} 88\right)$ was born in Calcutta, India, on October 16,1954. He received the B.Sc. (hons.) degree in physics, and the $B$.Tech. and M.Tech. degrees in radiophysics and electronics from the University of Calcutta. India, in 1974, 1977. and 1979. respectively. and the $\mathrm{Ph} . \mathrm{D}$. degree in electrical and computer en gineering from the University of California, Santa Barbara, in 1982

He was a Postdoctoral Fellow at the University of California. Santa Barabara, from September 1982 to February 1983. In March 1983 he joined the California Institute of Technology, Pasadena, as an Assistant Professor of Electrical Engineering, and is currently an Associate Professor in the same department. His main research interests are in digital signal processing, multirate filter bank systems, filter design, adaptive filtering, and multivariable system theory. He has been a consultant for several companies in California, in the areas of digital filtering. filter banks, and predictive coding.

Dr. Vaidyanathan served as the Vice-Chairman of the Technical Program Committee for the 1983 IEEE International Symposium on Circuits and Systems, and as an Associate Editor for the IEEE TRANSACTIONS ON CiRCUITS AND SYSTEMS for the period 1985-1987. He was a recipient of the Award for Excellence in Teaching at the California Institute of Technology, for the year 1983-1984. He is also a recipient of NSF's Presidential Young Investigator Award, starting from 1986. 